TECHNICAL REPORT 51

on

Contract NObs -65426

Index No. NS-200-021

TWO TUBE STEAM EVAPORATOR No. 3

W. Milich

E. A. Schultz

E. C. King

November 26, 1956

UJSA

MINE SAFETY APPLIANCES COMPANY

Callery, Pennsylvania 


\section{DISCLAIMER}

This report was prepared as an account of work sponsored by an agency of the United States Government. Neither the United States Government nor any agency Thereof, nor any of their employees, makes any warranty, express or implied, or assumes any legal liability or responsibility for the accuracy, completeness, or usefulness of any information, apparatus, product, or process disclosed, or represents that its use would not infringe privately owned rights. Reference herein to any specific commercial product, process, or service by trade name, trademark, manufacturer, or otherwise does not necessarily constitute or imply its endorsement, recommendation, or favoring by the United States Government or any agency thereof. The views and opinions of authors expressed herein do not necessarily state or reflect those of the United States Government or any agency thereof. 


\section{DISCLAIMER}

Portions of this document may be illegible in electronic image products. Images are produced from the best available original document. 

Copy No. 79

of 90 copies

File No. 1369

TECHNICAL REPORT $5 I$

on

Contract NObs-65426

Index No. NS-200-021

TWO TUBE STEAM EVAPORATOR NO. 3

W. Milich

E. A. Schultz

s. C. King

November 26, 1956
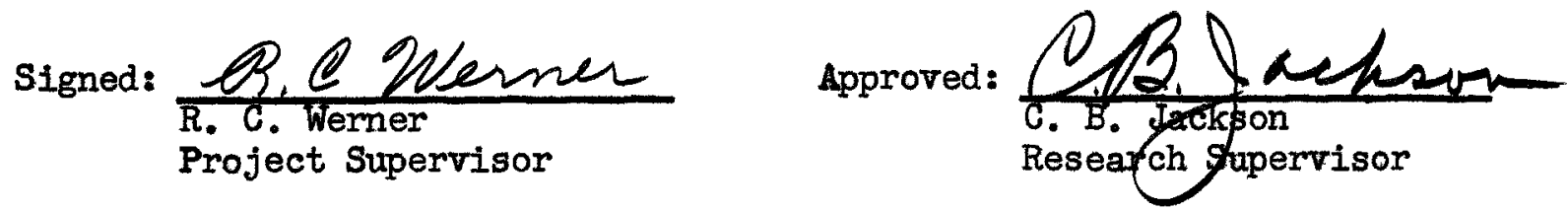

MINE SAFETY APPLIANCES COMPANY

Callery, Pa. 


\section{ABSTRACT}

1. INTRODUCTION 1

2. DESCRIPTION OF THE SYSTEM

2.1 Water-Steam System 3

2.2 NaK System 3

3. INITIAL STEADY STATE PROGRAM 6

3.1 Operation 6

3.2 Results - Steady State Condition $\quad 12$

4. CYCLIC PROGRAM 19

4.I Specifications 19

4.2 Operation 19

$\begin{array}{ll}4.3 \text { Results } & 19\end{array}$

5. FINAL STEADY STATE OPERATION 30

5.1 Operation 30

5.2 Results 30

6. EVAPORATOR WATER 33

7. OVERAIL RESULTS AND DISCUSSIONS 35

7.1 Comparison of Data on This Test 35

7.2 Comparison of This Evaporator With Two
Made of Stainless Steel

8. CONCLUSIONS 42 


\section{ABSTRACT}

A two tube steam evaporator made of $2 \frac{1}{4} \% \mathrm{Cr}-1 \% \mathrm{Mo}$ steel and having a gas-filled third fluid leak monitoring system (duplex tube), was tested and its performance compared with similar units constructed of types 304 and 347 stainless steel. Steam at 500 psig was generated, using a $\mathrm{NaK}$ inlet temperature of $850 \mathrm{~F}$.

The highest value of $765 \mathrm{Btu} / \mathrm{hr}-\mathrm{sq}$ ft- ${ }^{\circ} \mathrm{F}$ for the overall heat transfer coefficient, $J$, was obtained with a helium third fluid pressure of 200 psig. Lower coefficients were obtained at 20 and 600 psig. Using nitrogen, the optimum $U$ occurred with a third fluid pressure range between 20 and 200 psig.

At the start of the program the $U$ was found to be $660 \mathrm{Btu} / \mathrm{hr}-\mathrm{sq} \mathrm{ft-}{ }^{\circ} \mathrm{F}$ at a NaK flow of $2450 \mathrm{lbs} / \mathrm{hr}$-tube as compared to $610 \mathrm{Btu} / \mathrm{hr}-\mathrm{sq} \mathrm{ft-OF}$ at the completion of the program. This represents a $7 \frac{1}{2} \%$ drop in performance, with $75 \%$ of the drop occurring during the first $25 \%$ of the cyclic program. The overall drop in $U$ for the type 347 stainless steel evaporator was $2 \%$, and for the type 304 stainless steel evaporator was $37 \%$ before it failed.

The tubes in the present evaporator are to be reexpanded and the unit operated for an additional length of time to see if the original operating efficiency may be recovered. 
TWO TUBE STEAM EVAPORATOR NO. 3

\author{
W. Milich \\ E. A. Schultz \\ E. C. King \\ 1. INTRODUCTION
}

A problem of major concern in the liquid metal heating of steam generators has been the lack of integrity of the steam generating equipment. Most of the failures have been attributed to chloride stress corrosion of the stainless steel on the water side. While rigorous water treatment programs have been set up in order to extend the life of present equipment, it was proposed to investigate the possibility of using $2 \frac{1}{4} \% \mathrm{Cr}-$ 1\% Mo steel as a material of construction. One advantage offered by such a steel lies in its resistance to chloride stress corrosion.

This report covers the tests at Callery of a two duplex tube evaporator constructed of $2 \frac{1}{4} \% \mathrm{Cr}-1 \%$ Mo steel by the Griscom-Russell Company, Massillon, Ohio, under the supervision of Knolls Atomic Power Laboratory, Schenectady, New York and the Bureau of Ships, Department of the Navy, Washington, D. C. The test included water conditions similar to those of the B\&W Model Evaporator tests and both steady state and thermal cycling conditions as exemplified in the three major phases of the test program given below:

1. Operate the evaporator under steady state conditions prior to cycling to study the performance of the evaporator using first helium and then nitrogen as the third fluid medium in the duplex tube annuli.

2. Subject the evaporator to thermal cycling for a total of 11,200 cycles (10,000 regular cycles and 400 each of 3 special cycles using $\mathrm{N}_{2}$ as the third fluid).

3. Perform steady state operation of the evaporator, after completion of the thermal cycling program, at various power levels $\left(70,60,40,30,25\right.$ and $25 \%$ of full power using $\mathrm{N}_{2}$ as the third fluid).

The heat source used for this test was hot circulating NaK $(56 \% \mathrm{~K})$.

\title{
2. DESCRIPTION OF THE SYSTEM
}

A flow diagram of the entire system is shown in Fig. 1. Except for the evaporator, which is constructed of $2 \frac{1}{4} \% \mathrm{Cr}-1 \% \mathrm{Mo}$, all water and steam piping is of type 304 stainless steel. The $\mathrm{NaK}$ piping is 1 in. schedule 40, type 304 stainless steel. Transition welds from the evaporator to the stainless steel were made using Inconel "A" rod. 
AIR COOLED CONDENSERS

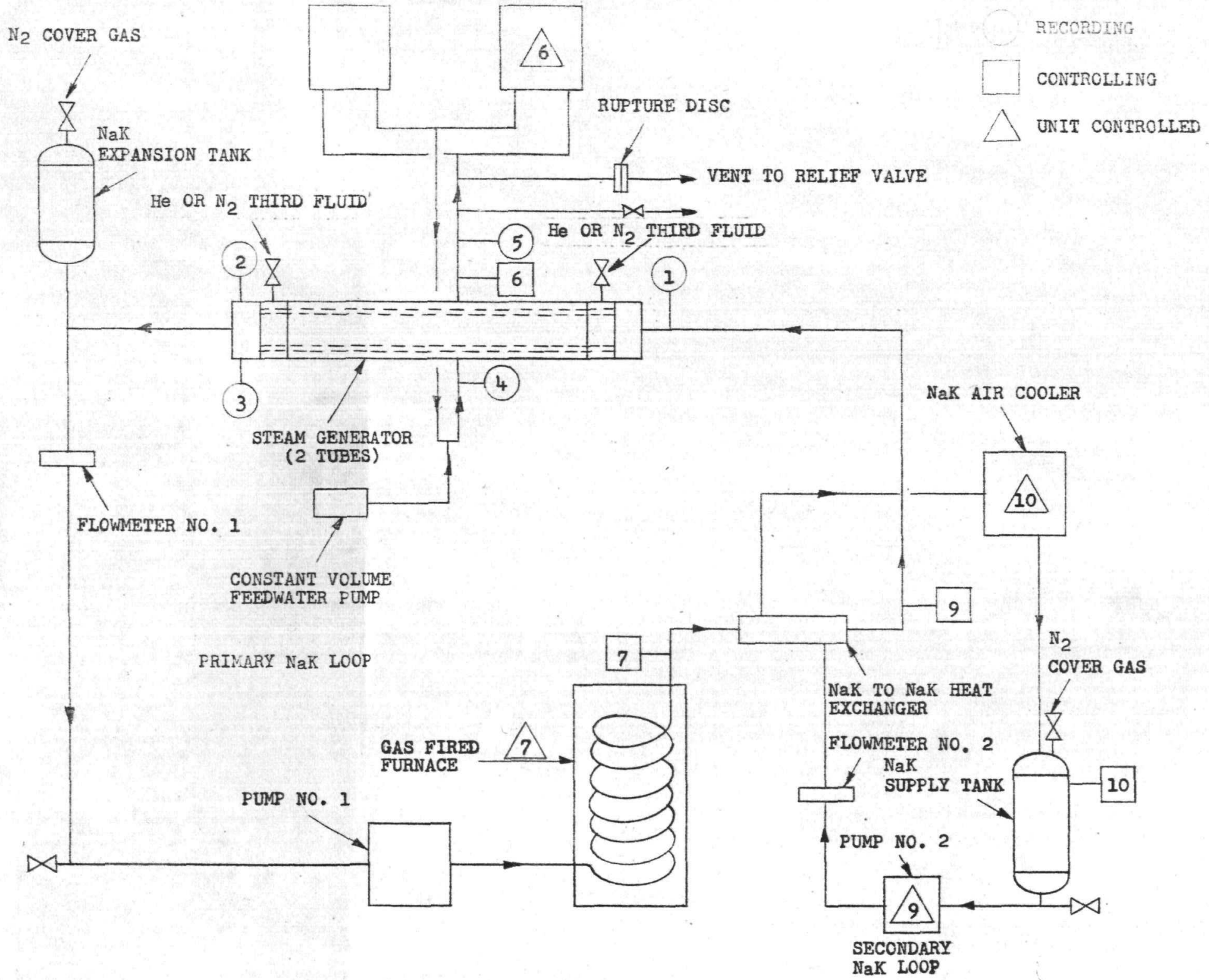

Fig. 1 - Flow Diagram - Duplex Two Tube Steam Generator System $\left(2 \frac{1}{4} \% \mathrm{Cr}-1 \% \mathrm{Mo}\right)$ 


\subsection{Water-Steam System:}

The evaporator, Fig. 2, contains two duplex tubes, initially bowed to handle thermal expansion. There are 36 grooves knurled into the outer surface of the inner tube, and equally spaced axially around the tube. When the inner tube was expanded into the outer tube to effect a mechanical bond between the tubes, the knurled grooves formed a third fluid leak detection system. Fig. 3, is a sketch showing tube to tube sheet details.

The pipe taps between the tube sheets are for introducing prepurified nitrogen or helium to the 36 grooves of the third fluid system, normally kept at a pressure intermediate between the steam and $\mathrm{NaK}$ pressures, to serve as a leak monitor.

The steam generated in the shell side of the evaporator (Fig. 1) flows through a 2 in. riser to two air cooled condensers; the condensate flows back to the evaporator by gravity. Provision was made for sampling the boiler water from the bottom of the evaporator.

The system was protected from excessive pressure buildup by a rupture disc sized for relief at 800 psig. A reseating safety valve down stream from the rupture disc served to maintain a back pressure should the rupture disc burst and thus prevent undue loss of water. An alarm was also installed to warn the operator if the steam temperature should fall outside prescribed operating limits.

\subsection{NaK Systems}

The liquid metal systems consisted of two independent NaK streams. The primary loop (Fig. 1) was used to transfer heat from a gas-fired furnace to the evaporator and the secondary loop was used to control the temperature of the primary $\mathrm{NaK}$ stream at the inlet to the evaporator, for cycling purposes, through the use of the double pipe heat exchanger. The secondary NaK stream was, in turn, air-cooled.

The NaK was pumped by an electromagnetic pump and the flow measured by a magnetic flowmeter. The $\mathrm{NaK}$ expansion and supply tanks were provided with pipe taps to introduce a cover gas such as prepurified grade nitrogen.

An alarm and automatic gas shut off to the furnace were incorporated in the system to protect the evaporator in case of loss of NaK flow or excessive $\mathrm{NaK}$ temperature. 


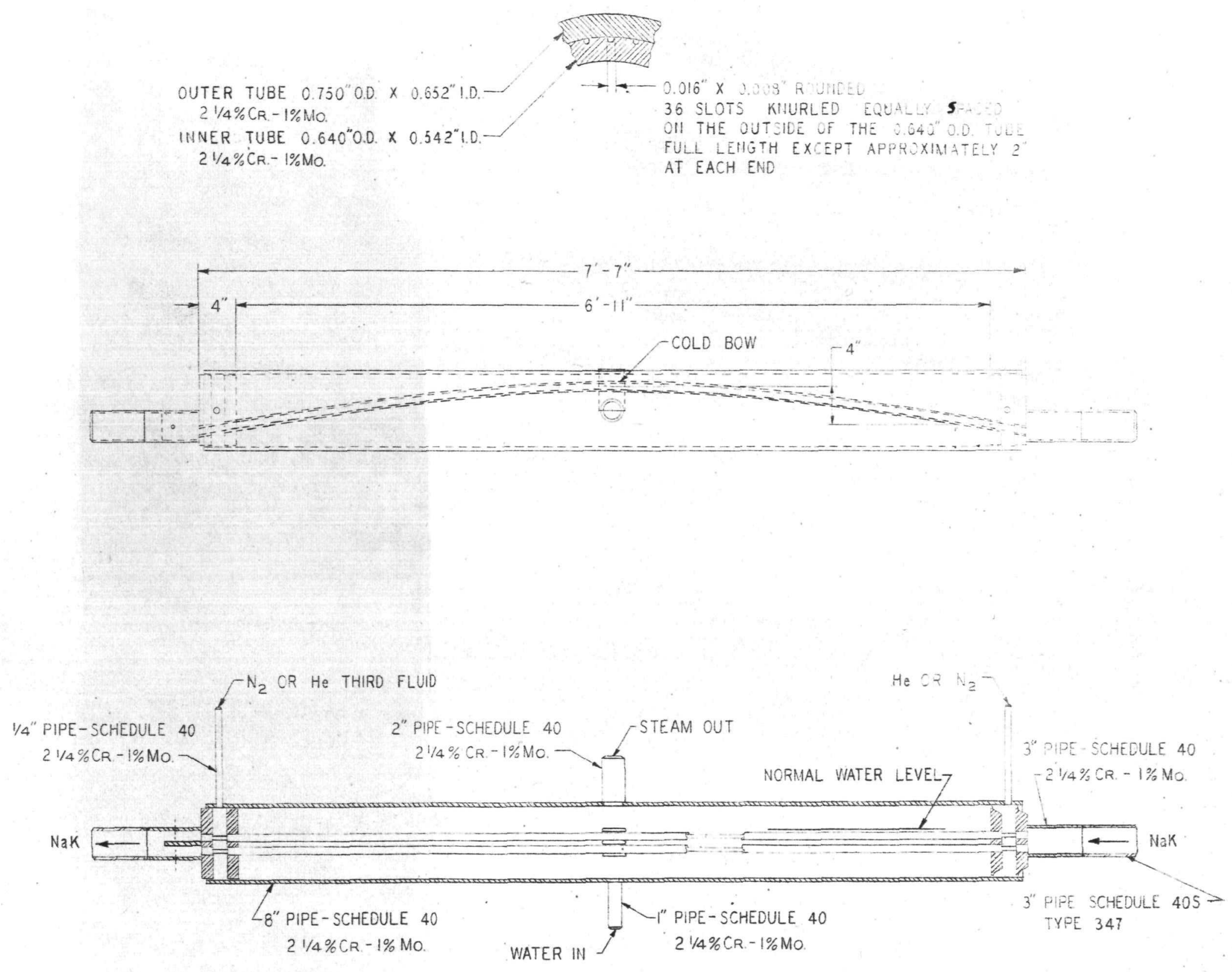

FIG 2 - TWO TUBE STEAM GENERATOR NO. 3 


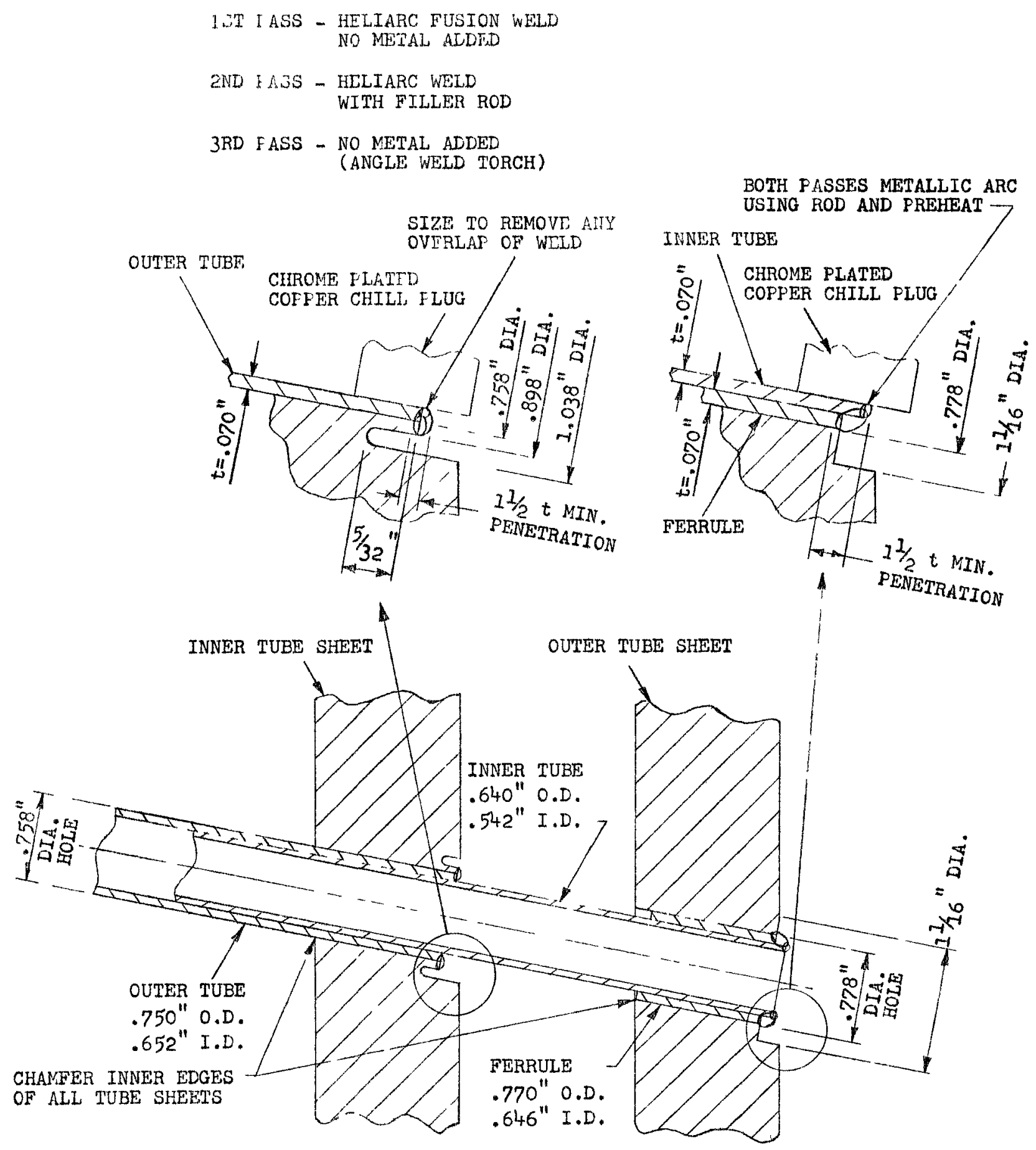

Fig. 3 - Detall of Tube Joints 


\section{INITIAL STEADY STATE PROGRAM}

\subsection{Operation}

At the start of the test program, steady state conditions were attempted, with $\mathrm{NaK}$ entering the evaporator at $850 \mathrm{~F}$, and the steam pressure maintained at 500 psig. The nitrogen pressure over the primary loop was kept at 50 psig, but only a slight positive pressure was imposed on the secondary loop. Nitrogen at $200 \mathrm{psig}$ was used as the third fluid. After four days of continuous operation at steady state conditions, the tubes in the evaporator were subjected to severe thermal shock when the main NaK pump was prematurely turned on after a power failure to the pump. As a result, the overall heat transfer coefficient, $U$, dropped from an original $650 \mathrm{Btu} / \mathrm{hr}-\mathrm{sq}$ ft- ${ }^{\circ} \mathrm{F}$ to $550 \mathrm{Btu} / \mathrm{hr}-\mathrm{sq} \mathrm{ft}-{ }^{\circ} \mathrm{F}$. After conferring with R. Mantey of KAPL (wire dated March 8, 1956) testing was stopped.

The evaporator was removed from the loop, washed, dried and leak tested with the mass spectrometer. No leak was evident. The evaporator was sealed and returned to Griscom-Russell Co., for retubing (March 12, 1956). In rebuilding the evaporator the following refinements in fabricating techniques were employed.

1. The outer surface of the inner tube was cleaned and polished prior to heat treatment and balt expansion. The cleaning and polishing removed any high spots on the tube surface caused by the knurling operation.

2. The heat treatment was performed in a hydrogen atmosphere to prevent the formation of an oxide film on the tube surfaces.

3. A larger diameter ball (0.558 in. as cormpared with 0.554 in. diameter) was used for expanding the rebuilt unit, resulting in a better mechanical bond.

4. Argon gas was circulated through the third fluid space during ball expansion of the rebuilt unit to help prevent oxidation.

With the rebuilt evaporator installed, steady state operation was resumed. On April 18, 1956 a pump failure resulted in loss of NaK flow. Natural circulation of the $\mathrm{NaK}$ caused high temperature transients at the inlet and outlet to the evaporator, Fig. 4. On May 22 a second high temperature incident occurred when the power was cut off during an electrical storm while at $40 \% \mathrm{NaK}$ flow. The plot of temperature vs time for this incident is found in Fig. 5.

Following these two incidents, the overall heat transfer coefficient had dropped about $2 \%$ which could be attributed to possible stress relieving of the mechanical bond between the tubes. The reason the damage (if any) to the bond from these later incidents was so slight as compared with the initial incident ( $2 \%$ vs $15 \%$ ) was because, in the first case, the hot slug of NaK was carried through the entire length of the tube. In,the latter two cases, the flow of hot $\mathrm{NaK}$ was by natural circulation only and was thereby only sufficient to maintain a large temperature difference a short distance into the evaporator before it was cooled by the boiling water. 


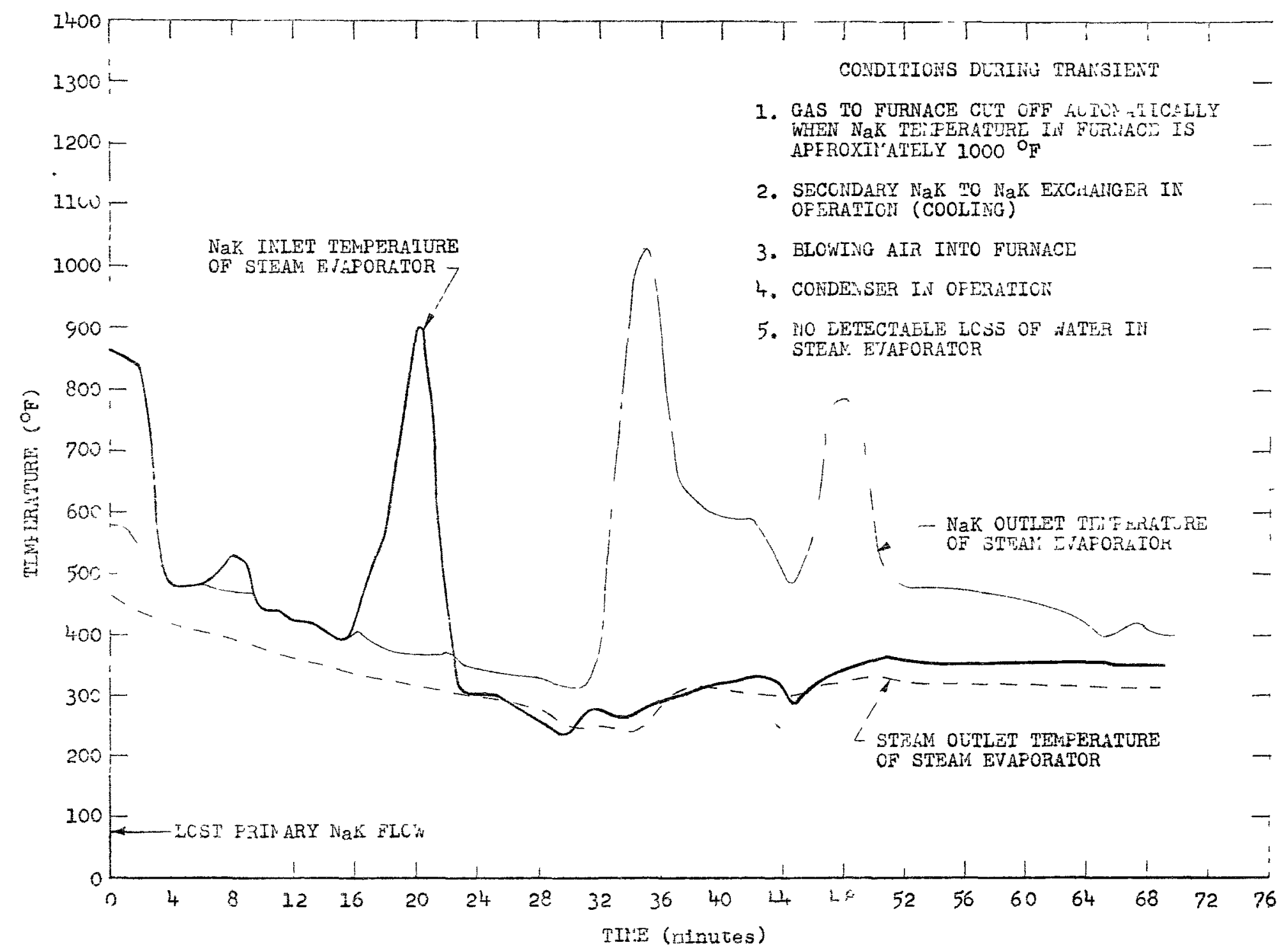

Fig. 4 - High MaK Temperature Incident $4-19-56$ During Operation 


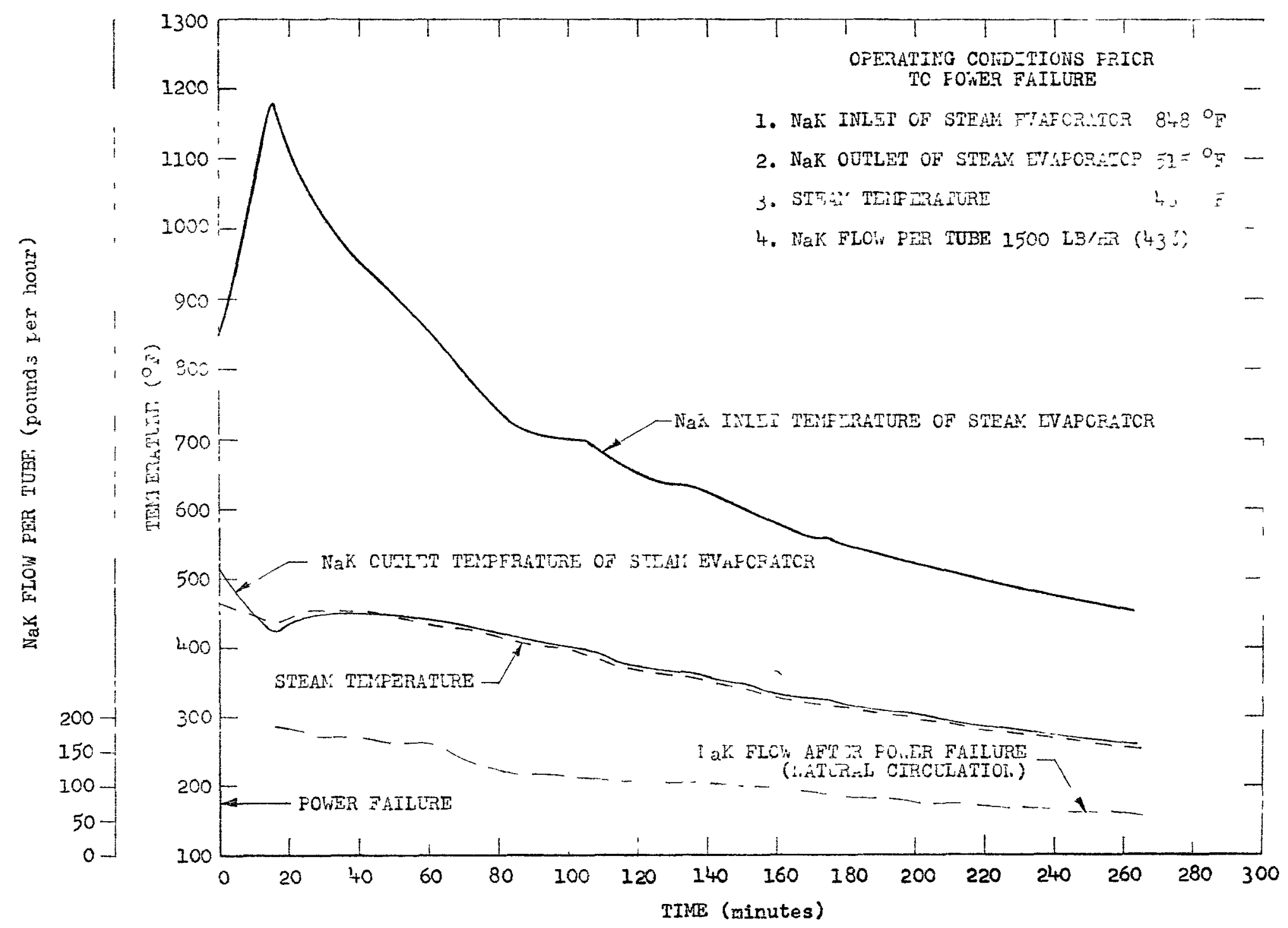

Flg. 5 - High NaK Terperature Transient Due to Fower Fallure 5-22-56 During Operation of $G-R$ Two Tube Evaporator No. 3 
It may be noted from Fig. 4 that both the inlet and outlet of the evaporator were subjected to hot $\mathrm{NaK}$. This incident resulted from loss of NaK flow but the NaK cooler remained in operation to cool the NaK. It was successful in reducing the maximum temperature but caused a flow reversal since it cooled the hot leg below the cold leg temperature. This phenomenon did not occur during power failure when flow through the NaK-NaK exchanger was also lost (see Fig. 5).

The chronological history for the steady state operating conditions is shown in Table 1 for the initial evaporator and in Table 2 after the evaporator had been repaired.

The maximum NaK operating conditions for the test apparatus were determined while operating at 500 psig steam pressure. The rated NaK flow for the evaporator had been set at $350 \mathrm{C}$ Ibs/hr-tube by KAPL. However, the maximum flow rate for these tests was established as $70 \%$ of $3500 \mathrm{lbs} / \mathrm{hr}$ tube or $2450 \mathrm{lbs} / \mathrm{hr}$-tube. This latter flow rate represented the maximum safe power level at which the equipment (furnace, pumps, etc.) could be operated continuously. During these tests an attempt was also made to correlate the variation of the heat transfer coefficient, $U$, with the pressure and nature of the third fluid gas, and with the rate of flow of $\mathrm{NaK}$. Helium and nitrogen were both used.

During all electrical storms, the evaporator power load was lowered to $30 \%$ to prevent damage to the unit, should a power failure occur while operating at the higher loads. 
Table 1 - Chronological History of Operation Prior to Rebuilding the Evaporator

\begin{tabular}{|c|c|}
\hline Date & Operation \\
\hline $\mathrm{Feb} .24$ & $\begin{array}{l}\text { During initial start up, the } 600-650 \text { psig blow out disc } \\
\text { prematurely ruptured at } 450 \text { psig. The relief valve failed } \\
\text { to reseat resulting in loss of } 2-3 \text { in. of water from the } \\
\text { evaporator. No damage inflicted to the evaporator. The } \\
\text { disc and relief valve were replaced and make up water ad- } \\
\text { ded to the boiler to operating level, ( } 1 \text { in. above top tube). }\end{array}$ \\
\hline Feb. 27 & $\begin{array}{l}\text { Operation resumed and after } 3 \text { hours of operation there was } \\
\text { an increase in system pressure to } 580 \text { psig which ruptured } \\
\text { the blowout disc rated } 623 \text { psig at } 575 \mathrm{~F} \text {. There was no } \\
\text { increase in steam temperature ( } 465 \mathrm{~F} \text { ) with this increase } \\
\text { in system pressure. The relief valve prevented undue } \\
\text { loss of the water. }\end{array}$ \\
\hline
\end{tabular}

Feb. 28 System examined to determine cause of pressure rise. Cause undetermined. Preparations made to resume operation.

Feb. 29 Operation resumed. Attempt made to reach rated temperature conditions.

Mar. 1 After 24 hours of operation the unit was shut down to inspect the pump and try to increase the primary NaK flow as the evaporator appeared to perform considerably beyond the predicted range. A $240 \mathrm{~F}$ temperature drop across the generator could not be majntained with a NaK inlet temperature of $850 \mathrm{~F}$. After over-hauling the pump, the system was again put into operation.

Mar. 2 - 7 Operation was continuous except as noted below. On 3/5/56 at 3:00 P.M. the primary NaK pump stopped for a short time due to overloading of the circuit breaker when attempting to increase the flow. The temperature of the NaK in the fumace rose to approximately $1500 \mathrm{~F}$ and, when the pump was prematurely turned on, a hot slug of $\mathrm{NaK}$ entered the evaporator. Operation was continued to study the damage of the shock to the heat transfer bond. On $3 / 6 / 56$ at $6: 00$ A.M. a small steam leak occurred in the water system on the return line to the evaporator near the boiler feed line from the pump. The unit was shut down for three hours while repairing the steam leak. Operation was then resumed. The unit was shut down on 3/7/56 for clean-up and returned to Griscom-Russell to be re-tubed. 
Table 2 - Chronological History of Operation

After Rebuilding the Evaporator

\begin{tabular}{ccc}
\hline Date & $\frac{\text { Operation }}{\text { April } 16-18}$ & Initial operations at steady state: \\
& NaK inlet to steam generator & $850 \mathrm{~F}$ \\
& Steam temperature & $467 \mathrm{~F}$ \\
& Nitrogen third fluid & $200 \mathrm{psig}$ \\
& NaK flow & $55,60,71 \%$
\end{tabular}

April 18(9:00 am-3:00 pm) Shut down to replace blow out disc.

April 18(3:00 pm-6:30 pm) Steady state at 70\% flow.

April $18(6: 30 \mathrm{pm})$ 21 (10:00 am)

April 21 - May 10

May $10-18$

May $18-20$

May 20

May $21-22$

May 22

May $22-29$

May $29-31$

Total Time: $\quad(4 / 16 / 56$ tp $5 / 31 / 56)$

Steaming - steady state operation

Cycling - trial cycles

Down time - repairs, etc. fluid. pressure.
Shut down - loss of NaK flow caused by bus bar dropping off at silver solder joint.

Steady State conditions:

Varied NaK flow 20 to $80 \%$ with variable nitrogen and helium third fluid pressures. (Vac. to 600 psig)

Shut down to repair steam leak and rebuild the $\mathrm{NaK}$ furnace which was badly cracked.

Steady state:

Varied NaK flow and helium pressure on third

Shut down to repair steam leak at T.C. weld.

Steady state conditions:

Varied NaK flow and helium third fluid

Down for 4 hours - power failure caused by electrical storm.

Steady state conditions:

Varied NaK flow and third fluid nitrogen and helium pressures.

Steady state at 30 and $70 \%$ flow, 200 psig $\mathrm{N}_{2}$, ran trial cycles. 


\subsection{Results - Steady State Conditions}

A summary of the results obtained on the evaporator prior to being rebuilt is found in Table 3, and after rebuilding in Table 4.

From data obtained during operation of the rebuilt evaporator, the overall heat transfer coefficient, $U$, was computed for various third fluid pressures, using first helium then nitrogen as the third fluid, Fig. 6. When using helium, the highest heat transfer coefficients calculated were at a third fluid pressure of $200 \mathrm{psig}$ indicating that the optimum pressure should be around the 200 psig. When nitrogen was used, the same values were obtained at third fluid pressures of 20 and 200 psig, indicating a maximum heat transfer rate between these two pressures.

Fig. 7 is a plot of the variation in overall heat transfer coefficient with variation in power level of the evaporator before the start of the thermal cycling program. The average value for the overall heat transfer coefficient at $30 \%$ power was about $480 \mathrm{Btu} / \mathrm{hr}-\mathrm{sq}$ ft- ${ }^{\circ} \mathrm{F}$, while at $70 \%$ power the overall coefficient was about $660 \mathrm{Btu} / \mathrm{hr}-\mathrm{sq}$ ft- ${ }^{\circ} \mathrm{F}$. 
Table 3 - Summary - Initial Steady State Results

\begin{tabular}{|c|c|c|c|c|c|c|c|c|c|c|c|c|c|}
\hline \multirow[b]{2}{*}{$\begin{array}{l}\text { Date } \\
1956 \\
\end{array}$} & \multicolumn{6}{|c|}{ Boiler Water Analyses } & \multicolumn{2}{|c|}{ NaK Tenp. ${ }^{\circ} \mathrm{F}$} & \multirow[b]{2}{*}{$\begin{array}{c}\text { Steam } \\
\text { Temp. } \\
\text { of } \\
\end{array}$} & \multirow{2}{*}{$\begin{array}{l}\text { NaK } \\
\text { Flow } \\
\text { lo } / \mathrm{hr}- \\
\text { tube } \\
\end{array}$} & \multirow[b]{2}{*}{$\mathrm{q}=\mathrm{Btu} / \mathrm{hr}$} & \multirow[b]{2}{*}{$\begin{array}{c}\mathrm{U} \\
\mathrm{Btw} / \mathrm{hr}- \\
\mathrm{sq} \mathrm{ft}-\mathrm{O}_{\mathrm{F}}\end{array}$} & \multirow[b]{2}{*}{ Coments } \\
\hline & $\underline{\mathrm{pH}}$ & $\begin{array}{l}\mathrm{PO}_{4}^{-3} \\
\mathrm{ppm} \\
\end{array}$ & $\begin{array}{l}\mathrm{SO}_{3}^{-2} \\
\mathrm{ppm}\end{array}$ & $\begin{array}{l}\mathrm{O}_{2} \\
\mathrm{ppm} \\
\end{array}$ & $\begin{array}{l}\mathrm{Cl}^{-} \\
\mathrm{ppm} \\
\end{array}$ & $\begin{array}{c}\text { Solids } \\
\text { ppm } \\
\end{array}$ & Inlet & Outlet & & & & & \\
\hline $2 / 27$ & 10.8 & 100 & 90 & $<0.05<$ & $<0.1$ & 583 & 689.8 & 509.7 & 462.0 & 2161.8 & 92,274 & 460.5 & $\begin{array}{l}\text { not at } \\
\text { equilibrium }\end{array}$ \\
\hline $2 / 29$ & 10.9 & 105 & 33 & $<0.05$ & & & 829 & 545 & 466.5 & 2362.9 & 159,434 & 623.3 & \\
\hline $3 / 1$ & 10.7 & 80 & 28 & $<0.05$ & & & 806.2 & 557.5 & 465.3 & 2759.9 & 166,597 & 653.8 & \\
\hline $3 / 2$ & & & & & & & 842.6 & 561.4 & 466.9 & 2791.0 & 186,005 & 663.7 & \\
\hline $3 / 3$ & & & & & & & 830.3 & 568.2 & 466.0 & 3040.2 & 188,851 & 665.7 & \\
\hline $3 / 4$ & 10.8 & 105 & 35 & $<0.05$ & & & 848.4 & 573.5 & 468.0 & 2027.8 & 197,265 & 668.8 & \\
\hline $3 / 5$ & 10.8 & 105 & 35 & $<0.05$ & & & 837.5 & 564.0 & 467.5 & 2878.2 & 186,562 & 666.7 & \\
\hline $3 / 5$ & 10.9 & 90 & 36 & $<0.05$ & & & 816.5 & 570.4 & 467.3 & 3239.2 & 188,653 & 681.0 & \\
\hline $3 / 5$ & & & & & & & 865.0 & 612.4 & 466.8 & 3295.7 & 196,053 & 567.2 & \\
\hline $3 / 6$ & & & & & & & 842.6 & 596.2 & 465.0 & 3046.5 & 177,906 & 555.7 & \\
\hline $3 / 7$ & 10.7 & 105 & 45 & $<0.05$ & & & 830.7 & 598.8 & 465.0 & 3214.7 & 176,680 & 556.8 & $\begin{array}{l}\text { shut down to } \\
\text { return } \\
\text { generator }\end{array}$ \\
\hline
\end{tabular}

\footnotetext{
' The NaK temperatures of the top tube and bottom tube were essentially the same.
} 
Table 4 - Steady State Heat Transfer Data on Two Tube Steam Generato No. 3,

Obtained During Initial Steady State Operation

\begin{tabular}{|c|c|c|c|c|c|c|c|c|c|}
\hline \multirow{3}{*}{$\begin{array}{l}\text { Date } \\
1956\end{array}$} & \multicolumn{2}{|c|}{ NaK Temp ${ }^{\circ} \mathrm{F}$} & \multicolumn{4}{|c|}{ NaK Flow } & \multirow{3}{*}{$\begin{array}{c}\mathrm{U} \\
\mathrm{Btu} / \mathrm{hr}- \\
\text { sq ft- }{ }^{\circ} \mathrm{F}\end{array}$} & \multirow{2}{*}{\multicolumn{2}{|c|}{$\begin{array}{l}\text { Third Fluid } \\
\text { Pressure-psig }\end{array}$}} \\
\hline & & & Steam & & $\%$ of 3500 & & & & \\
\hline & Inlet & Outlet & Temp $O_{F}$ & Ib/hr-tube & $\mathrm{Ib} / \mathrm{hr}$-tube & Btu/hr & & $\mathrm{N}_{2}$ & $\mathrm{He}$ \\
\hline $4 / 16$ & 857.8 & 520.9 & 465 & 1870 & 53.3 & 150,000 & 630 & 120 & \\
\hline & 871 & 523 & 464 & 1870 & 53.3 & 155.000 & 627 & 200 & \\
\hline $4 / 17$ & 867 & 527 & 464 & 1940 & 55.4 & 157,000 & 625 & 190 & \\
\hline & 866 & 530 & 465 & 1950 & 55.6 & 155,500 & 610 & 190 & \\
\hline & 850 & 535 & 465 & 2130 & 61.0 & 162.000 & 637 & 195 & \\
\hline & 852 & 534 & 465 & 2120 & 60.6 & 160,000 & 631 & 195 & \\
\hline & 850 & 548 & 467 & 2420 & 69.0 & 173,600 & 650 & 190 & \\
\hline & 855 & 548 & 467 & 2440 & 69.8 & 178,000 & 659 & 195 & \\
\hline & 851 & 548 & 467 & 2460 & 70.3 & 176,500 & 656 & 195 & \\
\hline & 854 & 549 & 466 & 2490 & 71.0 & 180,000 & 663 & 195 & \\
\hline $4 / 18$ & 856 & 551 & 465 & 2480 & 71.0 & 179,200 & 645 & 195 & \\
\hline & 867 & 571 & 466 & 2500 & 71.5 & 175,300 & 576 & 195 & \\
\hline $4 / 22$ & 826 & 528 & 465 & 1950 & 55.8 & 138,000 & 587 & 185 & \\
\hline $4 / 23$ & 809 & 525 & 463 & 1930 & 55.2 & 130,500 & 575 & 180 & \\
\hline & 871 & 551 & 464 & 2490 & 71.1 & 189,000 & 660 & 200 & \\
\hline & 854 & 550 & 464 & 2490 & 71.1 & 179,500 & 650 & 195 & \\
\hline & 838 & 548 & 465 & 2490 & 71.1 & 171,500 & $6 \longdiv { 4 6 }$ & 195 & \\
\hline & 846 & 550 & 465 & 2490 & 71.1 & 174,500 & 643 & 195 & \\
\hline & 855 & 551 & 465 & 2490 & 71.1 & 179,500 & 650 & 195 & \\
\hline & 847 & 561 & 465 & 2820 & 80.6 & 191,200 & 673 & 195 & \\
\hline & 848 & 561 & 465 & 2860 & 81.6 & 195,000 & 685 & 195 & \\
\hline & 847 & 562 & 465 & 2870 & 82.0 & 194,000 & 679 & 195 & \\
\hline & 850 & 566 & 465 & 2880 & 82.1 & 195,500 & 662 & 595 & \\
\hline & 850 & 566 & 465 & 2870 & 82.0 & 193,000 & 660 & 595 & \\
\hline & 851 & 567 & 465 & 2880 & 82.1 & 193,500 & 660 & 595 & \\
\hline & 856 & 588 & 465 & 2890 & 82.5 & 183,100 & 575 & 5 & $\mathrm{~mm} \mathrm{Hg}$ abs \\
\hline $4 / 24$ & 854 & 588 & 465 & 2870 & 82.0 & 180,500 & 570 & 5 & $\mathrm{~mm} \mathrm{Hg}$ abs \\
\hline & 855 & 588 & $4 6 \longdiv { 4 }$ & 2865 & 82.0 & 181,000 & 564 & 5 & $\mathrm{~mm} \mathrm{Hg}$ abs \\
\hline & 849 & 560 & 465 & 2830 & 80.8 & 193,800 & 681 & 195 & \\
\hline & 850 & 549 & 465 & 2460 & 70.3 & 175,000 & 645 & 200 & \\
\hline & $8 \longdiv { 4 0 }$ & 479 & 465 & 771 & 22.0 & 65,000 & 430 & 195 & \\
\hline & 845 & 475 & 464 & 692 & 19.8 & 62,500 & 434 & 195 & \\
\hline $4 / 25$ & 845 & 471 & 463 & 604 & 17.3 & 55,300 & 413 & 190 & \\
\hline & 852 & 473 & 464 & 604 & 17.3 & 55,000 & 397 & 180 & \\
\hline & 850 & 494 & 464 & 1115 & 31.9 & 94,800 & 496 & 185 & \\
\hline & 856 & 495 & 463 & 1120 & 32.0 & 97,000 & 488 & 200 & \\
\hline & 853 & 514 & 467 & 1470 & 42.0 & 118,700 & 537 & 200 & \\
\hline $4 / 26$ & 853 & 570 & 464 & 1440 & 41.0 & 118,000 & 533 & 190 & \\
\hline & 852 & 511 & $465^{\circ}$ & 1440 & 41.0 & 118,000 & 533 & 195 & \\
\hline & 855 & 496 & 463 & 1115 & 31.9 & 95,700 & 480 & 195 & \\
\hline & 848 & 496 & 465 & 1115 & 31.9 & 93,900 & 488 & 195 & \\
\hline & 844 & 499 & 465 & 1110 & 31.7 & 91,500 & 466 & 595 & \\
\hline & 845 & 498 & $4 6 \longdiv { 4 }$ & 1110 & 31.7 & 92,000 & 465 & 595 & \\
\hline & 855 & 500 & 465 & 1110 & 31.7 & 94,000 & 469 & 605 & \\
\hline
\end{tabular}


Table 4 - Steady State Heat Transfer Date on Two Tube Steam Generator No. 3,

Obtained During Initial Steady State Operation (continued)

\begin{tabular}{|c|c|c|c|c|c|c|c|c|c|}
\hline \multirow{2}{*}{$\begin{array}{l}\text { Date } \\
1956 \\
\end{array}$} & \multicolumn{2}{|c|}{ NaK Temp ${ }^{\circ} F$} & \multirow{2}{*}{$\begin{array}{c}\text { Steam } \\
\text { Temp }{ }^{\circ}\end{array}$} & $\mathrm{NaK} F$ & $\%$ of 3500 & \multirow{2}{*}{$\begin{array}{c}q= \\
\mathrm{Btu} / \mathrm{hr}\end{array}$} & \multirow{2}{*}{$\begin{array}{c}\mathrm{U} \\
\mathrm{Btu} / \mathrm{hr}- \\
\text { sq ft- } \mathrm{O}_{\mathrm{F}} \\
\end{array}$} & \multicolumn{2}{|c|}{$\begin{array}{l}\text { Third Fluid } \\
\text { Pressure-psig }\end{array}$} \\
\hline & Inlet & Outlet & & Ib/hr-tube & Ib/hr-tube & & & $\mathrm{N}_{2}$ & $\mathrm{He}$ \\
\hline $4 / 27$ & 864 & 497 & 464 & 1105 & 31.6 & 97,000 & 476 & 20 & \\
\hline & 853 & 496 & 464 & 1115 & 31.8 & 95,200 & 483 & 20 & \\
\hline & 857 & 496 & 464 & 1115 & 31.8 & 96,200 & 485 & 20 & \\
\hline & 852 & 496 & 464 & 1120 & 32.0 & $95^{\circ}, 300^{\circ}$ & 484 & 20 & \\
\hline & 851 & 524 & 465 & 1145 & 32.8 & 89,000 & 372 & 5 & $\mathrm{~mm} \mathrm{Hg}$ abs \\
\hline $4 / 28$ & 849 & 527 & 464 & 1165 & 33.3 & 89,000 & 362 & 5 & $\mathrm{~mm} \mathrm{Hg}$ abs \\
\hline & 849 & 528 & 464 & 1152 & 33.0 & 87,700 & 356 & 5 & $\mathrm{~mm} \mathrm{Hg}$ abs \\
\hline & 846 & 528 & 463 & 1160 & 33.1 & 87,500 & 354 & 5 & $\mathrm{~mm} \mathrm{Hg}$ abs \\
\hline & 846 & 528 & 465 & 1160 & 33.1 & 87,300 & 357 & 5 & $\mathrm{~mm} \mathrm{Hg}$ abs \\
\hline $4 / 29$ & 850 & 497 & 464 & 1160 & 33.1 & 97,700 & 495 & 190 & \\
\hline & $8 \longdiv { 4 8 }$ & 496 & 462 & 1160 & 33.1 & 97,000 & 486 & 190 & \\
\hline $4 / 30$ & 849 & 496 & 463 & 1150 & 32.8 & 97,000 & 492 & 185 & \\
\hline & 851 & 498 & 465 & 1160 & 33.1 & 97,700 & 494 & 190 & \\
\hline & 847 & 496 & 464 & 1130 & 32.3 & 95,000 & 488 & $195^{\circ}$ & \\
\hline & 855 & 525 & 465 & 1740 & 49.7 & 136,200 & 562 & 195 & \\
\hline & 854 & 524 & 465 & 1730 & 49.4 & 135,500 & 563 & 195 & \\
\hline & 849 & 523 & $465^{\prime}$ & 1740 & 49.6 & 134,500 & 568 & 195 & \\
\hline $5 / 1$ & 853 & 525 & 464 & 1780 & 51.0 & 138,000 & 570 & 200 & \\
\hline & 855 & 525 & 464 & 1780 & 51.0 & 139,500 & 571 & 200 & \\
\hline & 848 & 523 & 464 & 1740 & 49.7 & 134,200 & 562 & 200 & \\
\hline & 850 & 524 & 465 & 1735 & 49.6 & 134,200 & 560 & 200 & \\
\hline & 843 & 539 & 465 & 2118 & 60.4 & 152,500 & 595 & 200 & \\
\hline & 850 & 539 & 465 & 2100 & 60.0 & 155,000 & 600 & 200 & \\
\hline & 851 & 540 & 466 & 2105 & 60.1 & 155,500 & 598 & 200 & \\
\hline & 849 & 542 & 465 & 2110 & 60.2 & 153,500 & 583 & 595 & \\
\hline & 847 & 542 & 465 & 2100 & 60.0 & 152,000 & 577 & 595 & \\
\hline & 849 & 543 & 465 & 2120 & 60.5 & 153,900 & 583 & 590 & \\
\hline & 850 & 547 & 465 & 2160 & 61.6 & 158,200 & 604 & 35 & \\
\hline $5 / 2$ & 850 & 542 & 465 & 2180 & 62.2 & 159,300 & 605 & 20 & \\
\hline & 850 & 543 & 465 & 2165 & 61.8 & 158,000 & 597 & 30 & \\
\hline & 851 & 541 & 465 & 2110 & 60.3 & 155,100 & 592 & 20 & \\
\hline & 856 & 561 & 465 & 2102 & 60.1 & 147,100 & 507 & 5 & $\mathrm{~mm} \mathrm{Hg}$ abs \\
\hline & 850 & 561 & 464 & 2102 & 60.1 & 144,000 & 500 & 5 & $\mathrm{~mm}$ Hg abs \\
\hline & 851 & 566 & 464 & 2150 & 61.5 & 145,300 & 494 & 5 & $\mathrm{~mm}$ Hg abs \\
\hline $5 / 3$ & 851 & 568 & 465 & 2160 & 61.7 & 145,000 & 492 & 5 & $\mathrm{~mm}$ Hg abs \\
\hline & 853 & 565 & 465 & 2105 & 60.1 & 143,800 & 492 & 5 & m Hg abs \\
\hline & 844 & 537 & 465 & 2110 & 60.3 & 154,000 & 606 & 200 & \\
\hline & $845^{\circ}$ & 537 & 465 & 2100 & 60.0 & 153,600 & 602 & 200 & \\
\hline & 852 & 537 & 465 & 2909 & 59.8 & 156,200 & 605 & 200 & \\
\hline & 846 & 556 & $465^{\prime}$ & 2655 & 75.6 & 182,500 & 653 & 198 & \\
\hline & 843 & 557 & 466 & 2700 & 77.2 & 183,000 & 667 & 195 & \\
\hline $5 / 4$ & 844 & 556 & 465 & 2700 & 77.27 & 184,300 & 663 & 195 & \\
\hline & 844 & 555 & 465 & 2685 & 76.8 & 184,000 & 665 & 190 & \\
\hline & 850 & 557 & 466 & 2685 & 76.8 & 187,000 & 667 & 205 & \\
\hline & 848 & 558 & 465 & 2660 & 76.0 & 183,000 & 650 & 20 & \\
\hline & 850 & 558 & 465 & 2660 & 76.0 & 184,000 & 651 & 20 & \\
\hline & 848 & 560 & 464 & 2720 & 77.7 & 185,900 & 651 & 20 & \\
\hline
\end{tabular}


Table 4 - Steady State Heat Transfer Data on Two Tube

Steam Generator No. 3,

Obtained During Initial Steady State Operation

(continued)

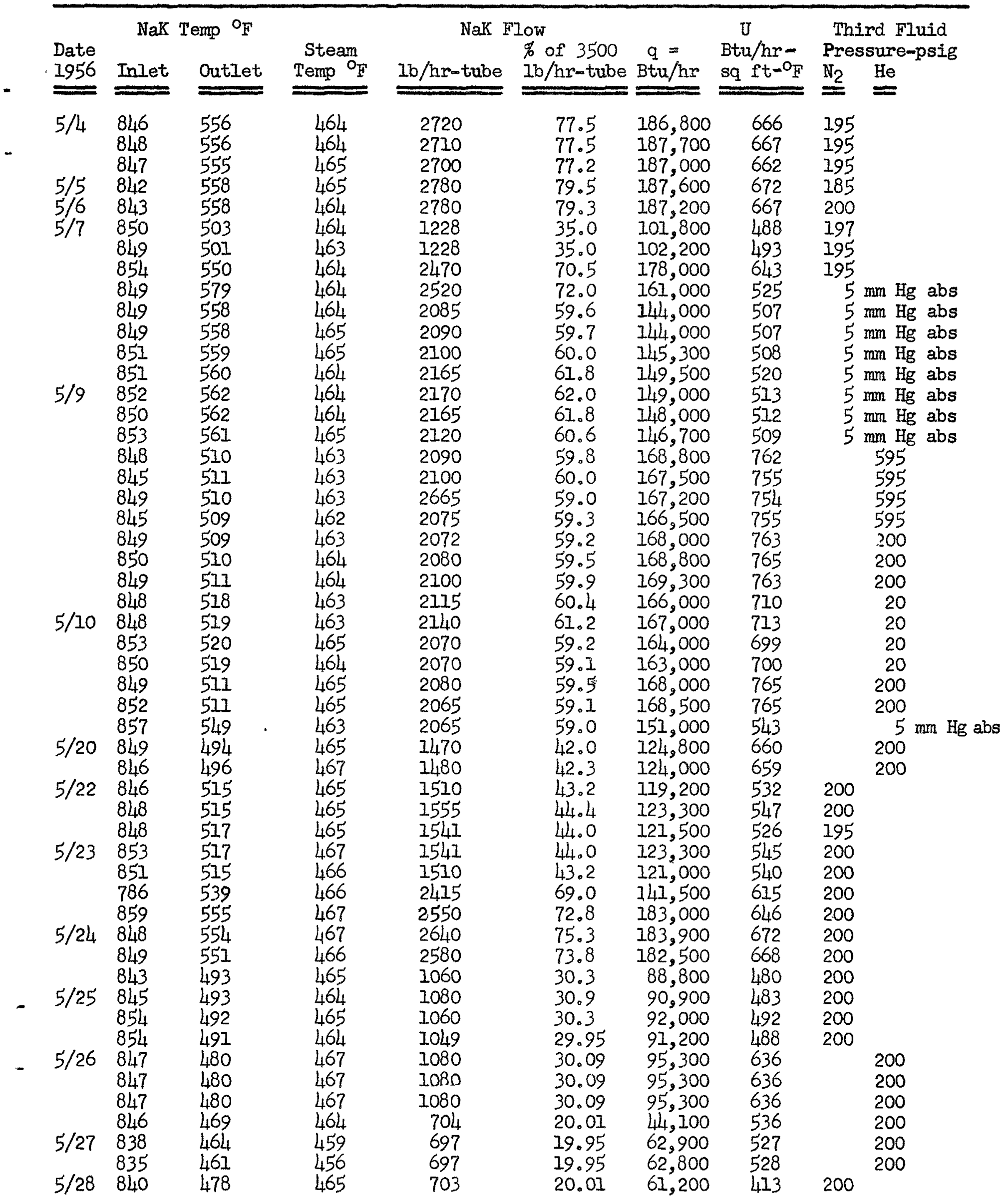




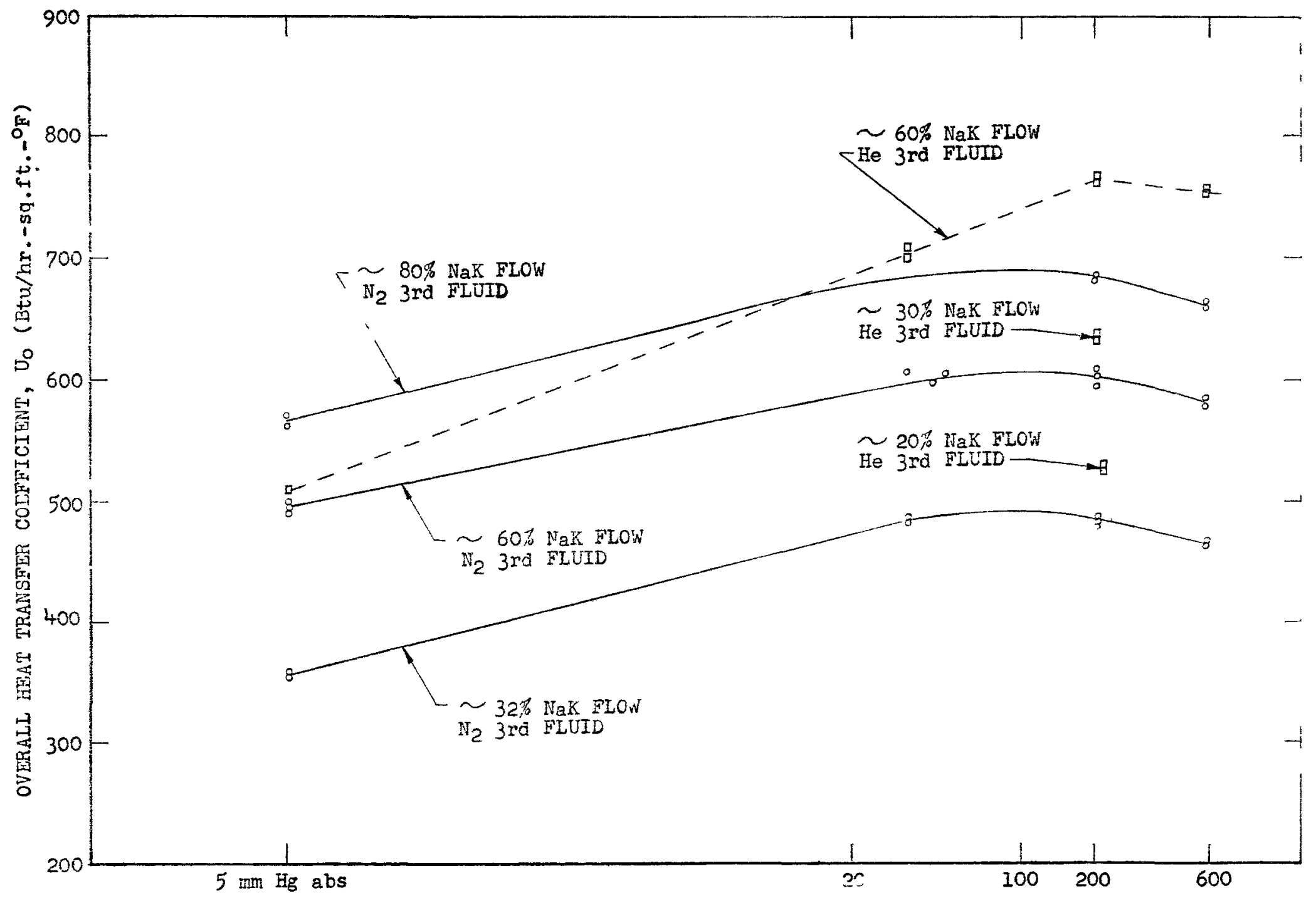

3rd FLUID FRESSURE (psig)

Fig. 6 - Overall Heat Transfer Vs. 3rd Fluid Fressure for G-R Two Tube Dvaporator No. 3 


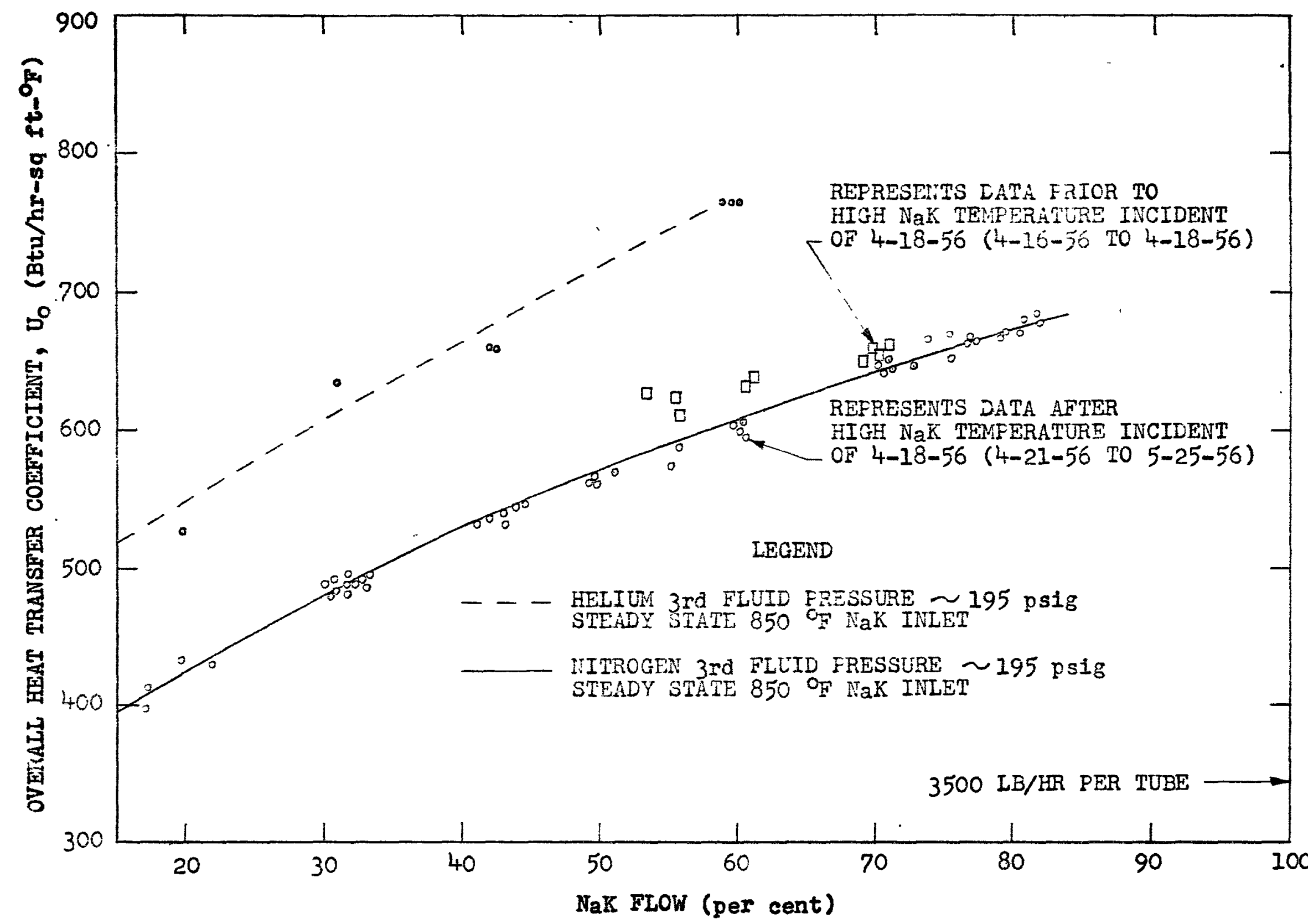

Fig. 7 - Heat Transfer Performance of Duplex Tube at Several Power Levels of G-R Two Tube Evaporator No. 3 


\section{GICLIC PROGRAM}

\subsection{Specifications}

The conditions for the cyclic program are tabulated in Table 5. At the start of the program 1600 standard (No. 2) cycles were to be run first. Then the three special cycles were to be introduced, running seven No. 2 cycles between each of the 3 special cycles.

\subsection{Operation}

Steady state heat transfer data was obtained daily by leveling out operations at $70 \%$ power and using a Leeds and Northrup precision potentiometer for thermocouple readings. A nitrogen third fluid pressure of 200 psig was used with 50 psig nitrogen cover gas. A chronological history of operation during the cyclic program is given in Table 6.

\subsection{Results}

Fig. 8 shows the change in overall heat transfer coefficient, $U$, as the cycling program progressed. At first, there was a rapid drop in $U$ from about 660 to about $630 \mathrm{Btu} / \mathrm{hr}-\mathrm{sq}$ ft-oF when the special thermal cycles were introduced. The further drop in $\mathrm{J}$ to about $610 \mathrm{Btu} / \mathrm{hr}-\mathrm{sq} \mathrm{ft-OF}$ over the remainder of the cycling program was much more gradual.

Plots of conditions typical of each of the four thermal cycles used in the program are shown in Fig. 9, 10, 11 and 12. Fig. 13, is a typical 24 hour chart of the $\mathrm{NaK}$ temperature at the evaporator inlet during the cycling program. A tabulation of the steady state heat transfer data obtained after the start of the thermal cycling program is shown in Table 7 .

On June 19, a faulty relay caused a short circuit and loss of power, at the top of a standard (No. 2) cycle. The plot of $\mathrm{NaK}$ temperature and steam temperatures within the evaporator during this high temperature excursion is found in Fig. 14. This incident occurred just at the time of the introduction of the special thermal cycles (No. 1, 3 and 4) to the cycling program. Since the slope of the curve of $U$ vs cycle numbers immediately before and after the power failure did not change, and since shortly thereafter the slope did decrease, it is difficult to state conclusively that any possible damage to the unit was attributable to this incident or to the co-current special thermal shock program. 
Table 5 - Gyclic Program for Two Tube Steam Evaporator No. 3

\begin{tabular}{|c|c|c|c|c|c|c|}
\hline $\begin{array}{l}\text { Cycle } \\
\text { No. }\end{array}$ & $\begin{array}{l}\text { Inlet } \mathrm{NaK} \\
\text { Temp. } \\
\text { (OF) }\end{array}$ & $\begin{array}{l}\text { Time } \\
\text { Seauences } \\
\text { (Sec) }\end{array}$ & $\begin{array}{c}\text { NaK Flow } \\
\% \text { of } \\
3500 \mathrm{lb} / \mathrm{hr}-t u b e\end{array}$ & $\begin{array}{l}\text { Steam } \\
\text { Pressure } \\
\text { Psig } \\
\end{array}$ & $\begin{array}{l}\text { Cycles } \\
\text { Required }\end{array}$ & Method of Control \\
\hline 1 & $\begin{array}{l}850 \\
946 \\
850\end{array}$ & $\begin{array}{l}\text { Balance } \\
30 \text { up } \\
300 \text { hold } \\
300 \text { down } \\
\text { Balance }\end{array}$ & 70 & 500 & 400 & $\begin{array}{l}\text { Reduce primary NaK } \\
\text { flow to } \sim 43 \% \text {. Reduce } \\
\text { secondary loop flow to } \\
\text { minimum steady flow. }\end{array}$ \\
\hline 2 & $\begin{array}{l}850 \\
740 \\
850\end{array}$ & $\begin{array}{l}30 \text { down } \\
180 \text { hold } \\
30 \text { up } \\
180 \text { hold }\end{array}$ & 70 & 500 & 10,000 & $\begin{array}{l}\text { Primary NaK flow re- } \\
\text { duced to } \sim 28 \% \text {. Cool } \\
\text { with secondary } \mathrm{NaK} \\
\text { loop: }\end{array}$ \\
\hline 3 & $\begin{array}{r}850 \\
1050 \\
850\end{array}$ & $\begin{array}{l}\text { Balance } \\
120 \text { up } \\
300 \text { hold } \\
300 \text { down } \\
\text { Balance }\end{array}$ & As necessary & 500 & 400 & $\begin{array}{l}\text { Primary NaK flow re- } \\
\text { duced to } \sim 27 \% \text {. Sec- } \\
\text { ondary NaK loop at } \\
\text { minimum flow. }\end{array}$ \\
\hline 4 & $\begin{array}{l}850 \\
650 \\
850\end{array}$ & $\begin{array}{l}\text { Balance } \\
2 \text { down } \\
300 \text { hold } \\
300 \text { up } \\
\text { Balance }\end{array}$ & 70 & 500 & 400 & $\begin{array}{l}\text { Primary NaK flow con- } \\
\text { stant at } \sim 68.8 \% \text {. Cool } \\
\text { with secondary NaK loop. }\end{array}$ \\
\hline
\end{tabular}


Table 6 - Chronological History of Two

Tube Steam Generator No. 3

During Cycling Program

\begin{tabular}{|c|c|}
\hline Jate & Operation \\
\hline June $1-3$ & $\begin{array}{l}\text { Cycling Condition } 2 \text { - Daily heat transfer } \sim 70, \\
\text { steadv state }-1 \mathrm{hr} \text {. }\end{array}$ \\
\hline June $11-5$ & $\begin{array}{l}\text { Replace siyht glass - } 70 \% \text { steady state, cycling, } \\
30 \% \text { steady state. }\end{array}$ \\
\hline June $6-20$ & $\begin{array}{l}\text { Gycling Condition } 2 \text { - at } 70 \% \text { steady state for heat } \\
\text { transfer and } 30 \% \text { during storms. liade adjustments } \\
\text { for special cyclic conditions } 1,3 \text { and } 4 \text {. }\end{array}$ \\
\hline June $21-30$ & $\begin{array}{l}\text { Special cycles introduced running a series of } 7 \text { No. } 2 \\
\text { cycles between each No. } 1,3 \text { and } 4 \text { cycle. Ran } 70, \% \\
\text { steady state to adjust instruments, and } 30 \% \text { during } \\
\text { storms. }\end{array}$ \\
\hline July $1-3$ & $\begin{array}{l}\text { Down for repair of Secondary NaK exchanger. Snall } \\
\text { secondary Nair to air leak at bellows. New charce of } \\
\text { water added to boiler. }\end{array}$ \\
\hline JuJy $4-11$ & $\begin{array}{l}\text { Back in operation and cycling except whon ocessary } \\
\text { to be at } 70^{\circ} \text { steady state during heat tr irfer read- } \\
\text { ings and adjustments in instrumentation. operation at } \\
30^{\circ} \text { during storms. }\end{array}$ \\
\hline JuIy $12-13$ & $\begin{array}{l}\text { Down for repair of secondary NaK exchanter. Small } \\
\text { secondary NaK leak to air at bellows again. Rede- } \\
\text { signed exchanger with bellows at cold } \text { nd with protec- } \\
\text { tive sleeve and shroud. }\end{array}$ \\
\hline July $14-15$ & $\begin{array}{l}\text { Gycling except for } 70,6 \text { operation for adjustments and } \\
\text { heat transfer. }\end{array}$ \\
\hline Julv $16-20$ & $\begin{array}{l}\text { Steady state operation at } 70 \text { and } 30 \% \text { flows. Repairing } \\
\text { automatic cycling instrumentation. }\end{array}$ \\
\hline July $21-31$ & $\begin{array}{l}\text { Cycling operations except } 70 \% \text { daily heat transfer and } \\
\text { adjustments. Operation at } 30 \% \text { ruring storms. }\end{array}$ \\
\hline Aug. $1-7$ & $\begin{array}{l}\text { Cycling, except for } 70 \% \text { operation for daily heat } \\
\text { transfer and } 30 \% \text { for storms. }\end{array}$ \\
\hline Aug. 8 & Down for repair of promix bearings. \\
\hline Aug. $9-17$ & $\begin{array}{l}\text { Cycling, except for } 70 \% \text { oporation for daily heat } \\
\text { transfer and } 30,0 \text { operation during storms. }\end{array}$ \\
\hline Aug. $18-25$ & $\begin{array}{l}\text { Dow for repair of Nak leak in seconrary exchanger. } \\
\text { Now exchanger installed ceaturing hairpin design to } \\
\text { elininate bellows. }\end{array}$ \\
\hline $\begin{array}{l}\text { Aug. } 26,- \\
\text { sept. } 9\end{array}$ & $\begin{array}{l}\text { Cycling except for adjustments to instrumentation. } \\
\text { 70\% operation for dajiy lieat transfer and } 30 \% \text { operation } \\
\text { during storms. }\end{array}$ \\
\hline Sept. 9 & $\begin{array}{l}\text { Completed 10,000 stanitard cycles and } 400 \text { each of the } \\
\text { special cycles. Ind of cycling program. }\end{array}$ \\
\hline
\end{tabular}




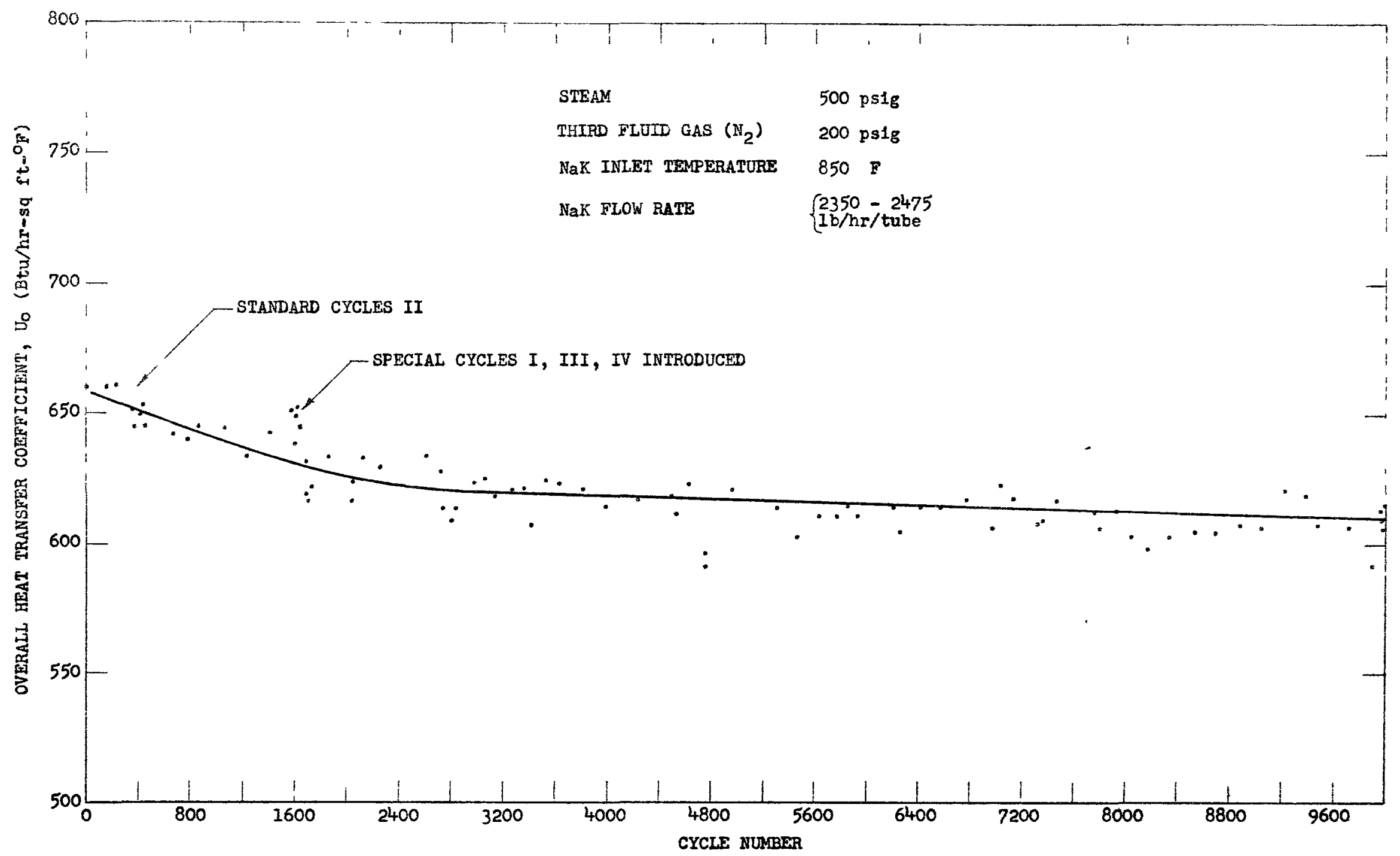

F1g. 8 - Heat Transfer Performance of Griscom-Russell Two. Tube Steam Evaporator No. 3 

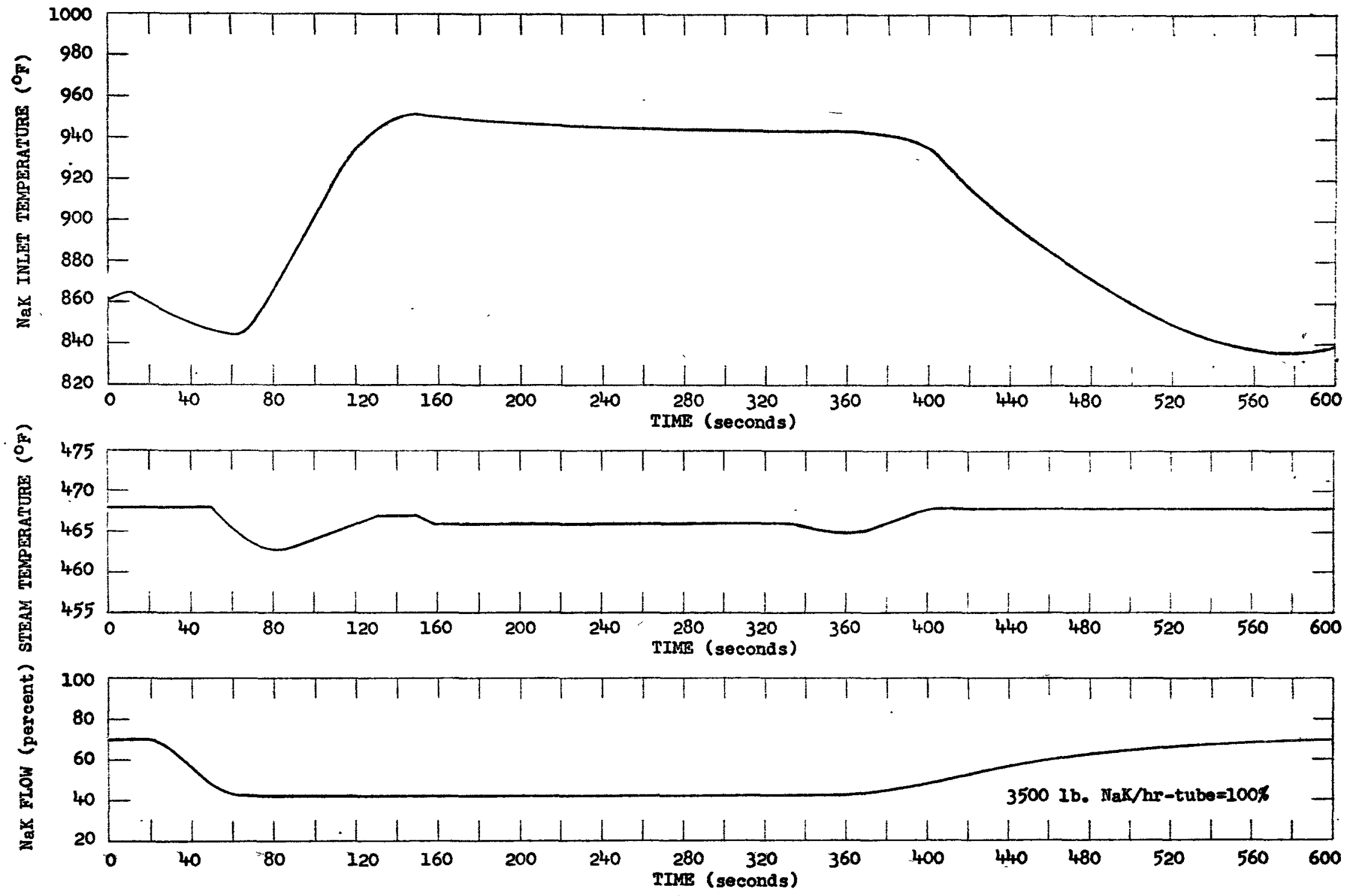

Fig. 9 - Cyclic Condition I of Griscom-Russell Two Tube Steam Eraporator No. 3 

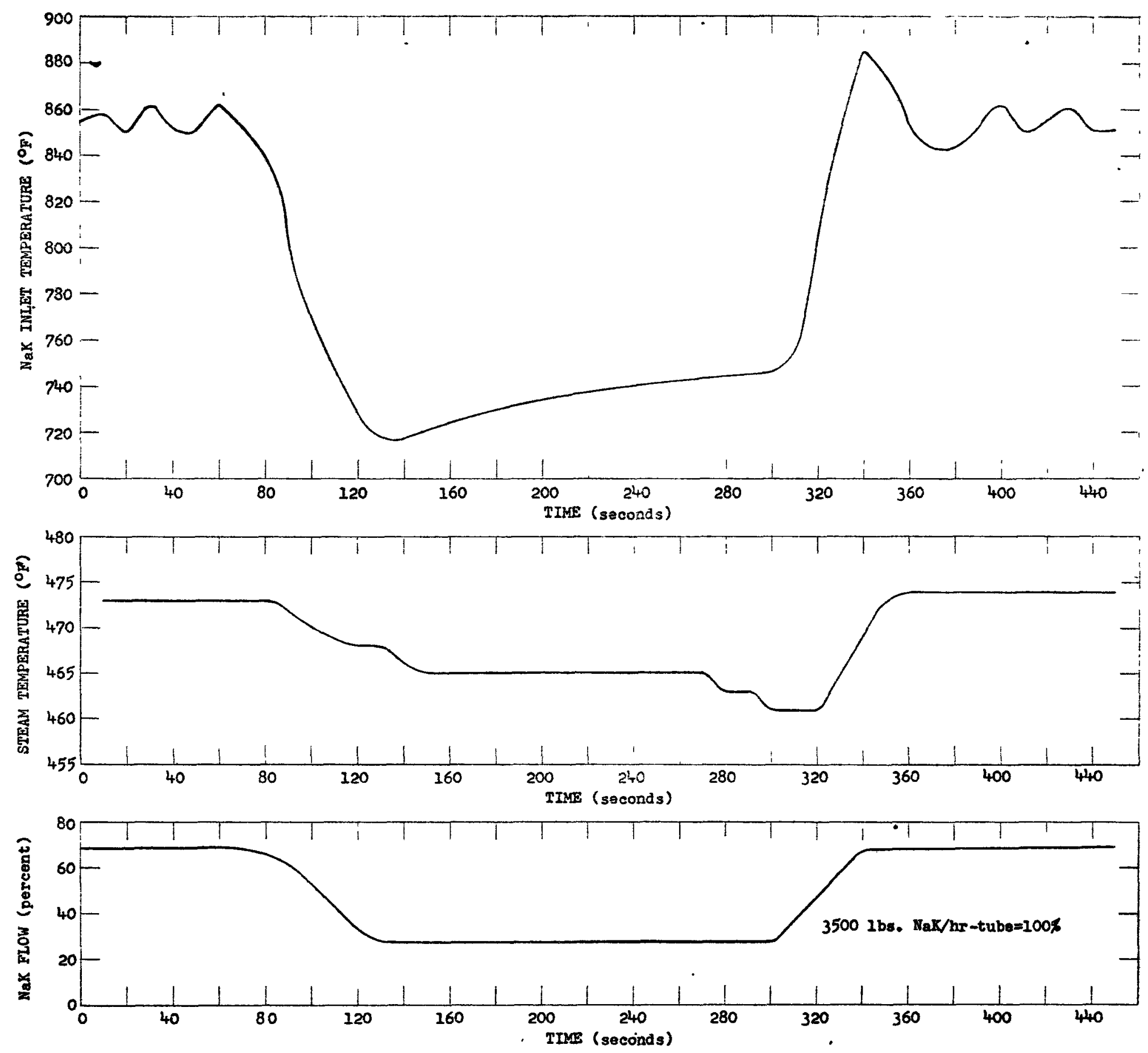

Fig. 10 - Cyellc Conation II of Criscom-Russell Two Tube Steam Bvaporator No. 3 

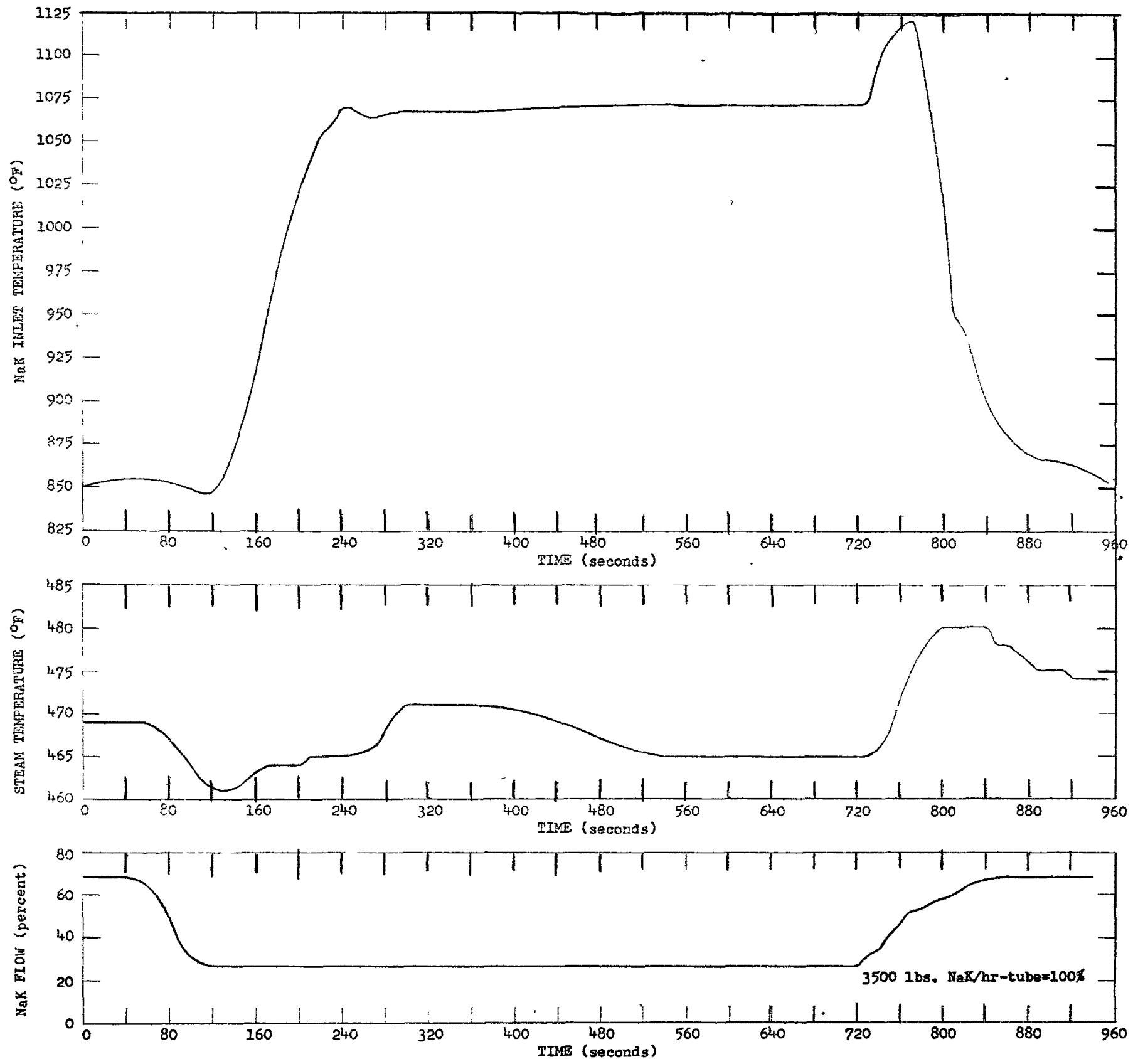

Fig. 11 - Cycilc Condition III of Griscom-Russell Two Tube Steam Evaporator No. 3 

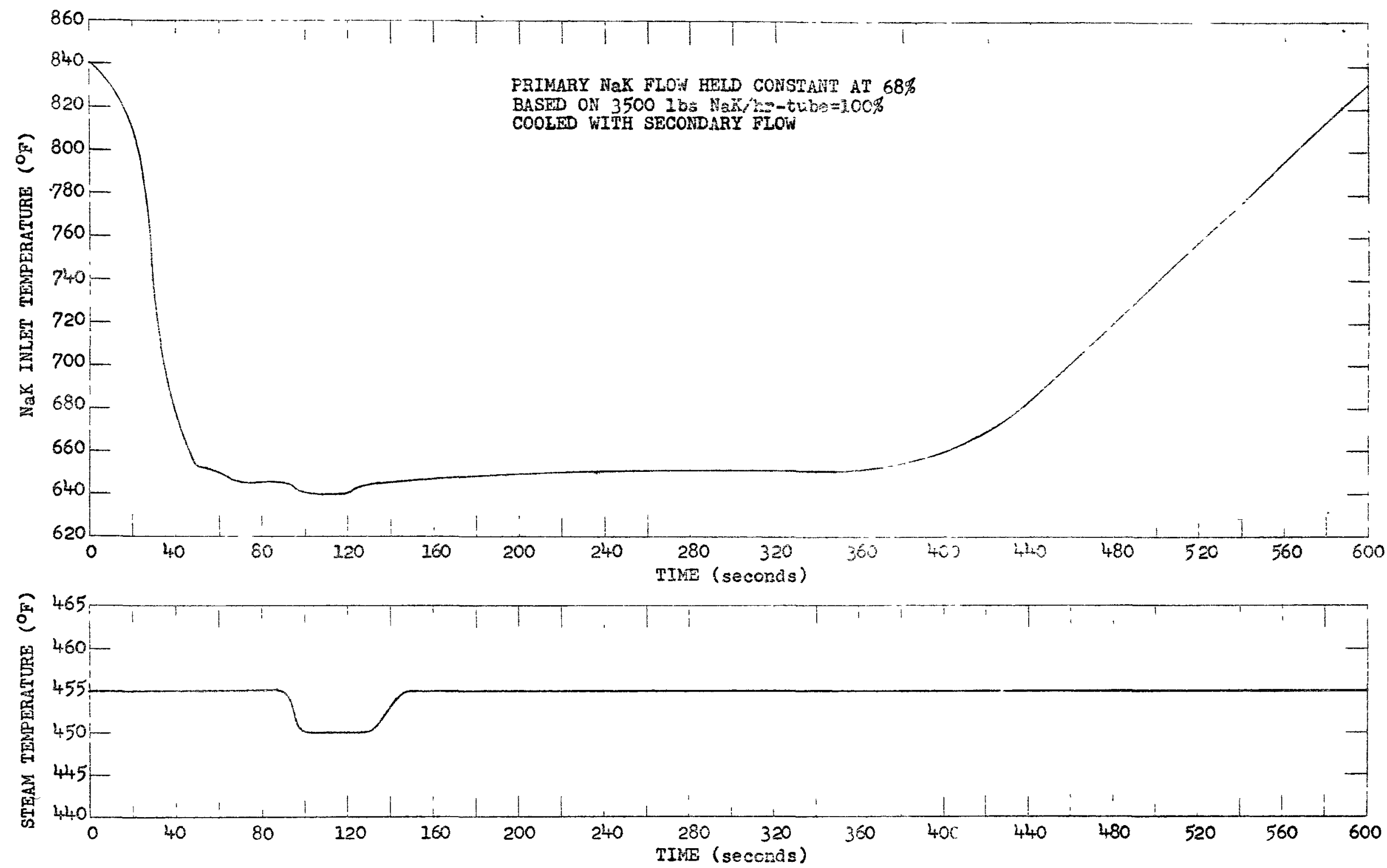

Fig. 12 - Cyclic Condition IV of Griscom-Russell Two Tube Steal Evaporator No. 3 


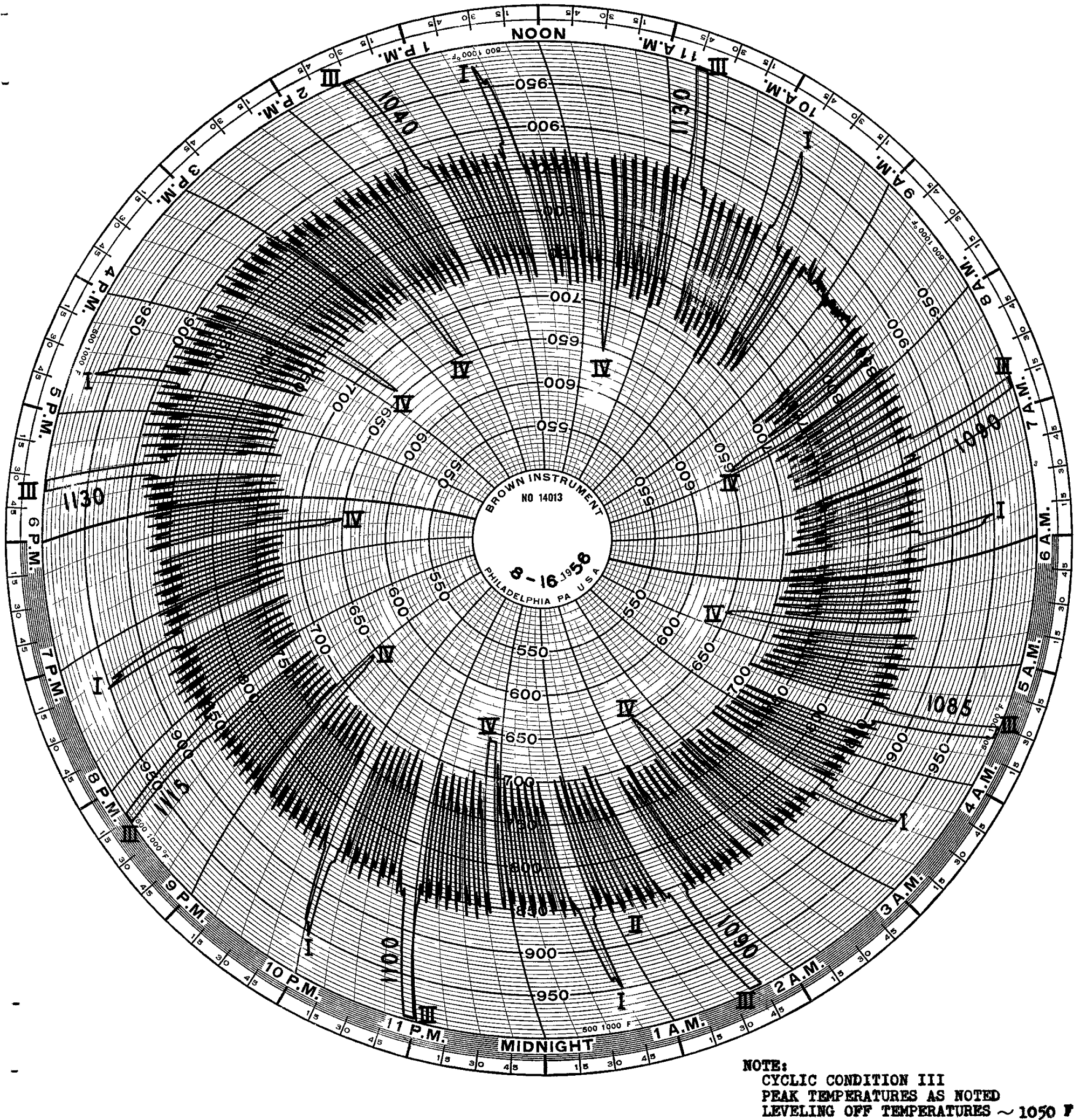

Fig. 13 - Chart Showing NaK Inlet Temperature to Steam Generator Mo. 3 
Table 7 - Hest, Tranafer Steadv State Tost Data (during cyciling progreni)

Two Tubs Fvaporator No. 3, (200 paig Hitrogen Third Fluid)
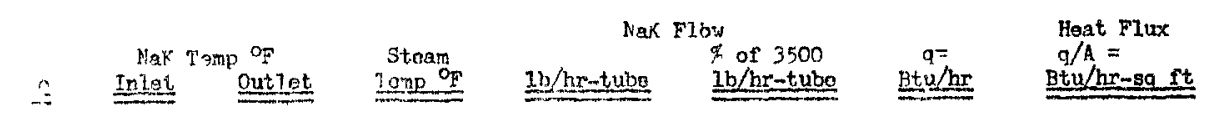

\begin{tabular}{cc}
$\begin{array}{c}0 \\
\text { Btu/hr- } \\
\text { sq ft-oF }\end{array}$ & Cycle \\
\hline
\end{tabular}

\begin{tabular}{|c|c|c|}
\hline 3 & 835 & 542 \\
\hline & 644 & 545 \\
\hline $1 / 5$ & $850^{\circ}$ & 548 \\
\hline & 856 & 545 \\
\hline 16 & $85 n$ & 544 \\
\hline 17 & 850 & 545 \\
\hline 18 & 853 & 545 \\
\hline $1 / a$ & 855 & 545 \\
\hline 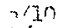 & 872 & 542 \\
\hline $6 \sqrt{11}$ & 824 & 540 \\
\hline$/ 12$ & 851 & 550 \\
\hline $6 / 13$ & 854 & 545 \\
\hline & 850 & 546 \\
\hline & 850 & 501 \\
\hline $6 / 15$ & 854 & 547 \\
\hline$D / 16$ & 846 & 543 \\
\hline $6 / 17$ & 850 & 547 \\
\hline $6 / 18$ & 846 & 540 \\
\hline $6 / 19$ & 848 & 550 \\
\hline $6 / 20$ & 849 & 551 \\
\hline & 850 & 550 \\
\hline & 853 & 553 \\
\hline & 832 & 550 \\
\hline $0 / 20$ & 851 & 501 \\
\hline$b /<1$ & 840 & $\begin{array}{l}4 / 4 \\
547\end{array}$ \\
\hline $6 / 2 ?$ & 852 & 552 \\
\hline $0 / 25$ & 850 & 552 \\
\hline $6 / 26$ & 849 & 552 \\
\hline $5 / 27$ & 847 & 547 \\
\hline $6 / 28$ & 561 & 553 \\
\hline $6 / 20$ & 853 & 553 \\
\hline $7 / 4$ & 851 & 554 \\
\hline $7 / 5$ & 849 & 545 \\
\hline $7 / 5$ & 849 & 552 \\
\hline $7 / 6$ & 850 & 554 \\
\hline $7 / 6$ & $\$ 52$ & 552 \\
\hline $7 / 7$ & 851 & 554 \\
\hline $7 / 8$ & 861 & 558 \\
\hline $7 / 9$ & 858 & 551 \\
\hline $7 / 10$ & 855 & 550 \\
\hline $7 / 1 / 4$ & 855 & 557 \\
\hline $7 / 20$ & 833 & 549 \\
\hline $7 / 21$ & 854 & 555 \\
\hline $7 / 22$ & 848 & $55 ?$ \\
\hline $7 / 23$ & 856 & 556 \\
\hline $7 / 24$ & 850 & 553 \\
\hline $7 / 25$ & 849 & 553 \\
\hline $7 / 26$ & 858 & 554 \\
\hline $7^{\prime} 27$ & 857 & 547 \\
\hline $7 / 28$ & 850 & 549 \\
\hline $7 / 29$ & 851 & 549 \\
\hline $7 / 30$ & 847 & 552 \\
\hline $7 / 31$ & 848 & 549 \\
\hline $8 / 1$ & 853 & $55 ?$ \\
\hline $8 / 2$ & $85 ?$ & 552 \\
\hline $8 / 3$ & 848 & 550 \\
\hline $8 / l_{4}$ & 847 & 550 \\
\hline $8 / 6$ & 856 & 550 \\
\hline$B / 7$ & 755 & $55 ?$ \\
\hline $8 / 10$ & 853 & 553 \\
\hline $8 / 11$ & 857 & 553 \\
\hline $8 / 22$ & 844 & 552 \\
\hline $8 / 13$ & 8149 & 551 \\
\hline $3 / 14$ & 848 & 553 \\
\hline $8 / 15$ & 248 & 351 \\
\hline $8 / 16$ & 849 & $=52$ \\
\hline $8 / 17$ & 851 & $55]$ \\
\hline $8 / 26$ & 844 & 555 \\
\hline $8 / 27$ & $85 ?$ & 253 \\
\hline $8 / 2 \overrightarrow{2}$ & 844 & 554 \\
\hline$B / 29$ & $8 / 44$ & 554 \\
\hline $8 / 30$ & 848 & 5,3 \\
\hline $8 / 31$ & 852.3 & 556 \\
\hline $9 / 1$ & $8,4,8$ & 35 \\
\hline $9 / 2$ & 850 & 556 \\
\hline $9 / 3$ & 848 & 351 \\
\hline $9 / 4$ & 850 & $54^{\circ}$ \\
\hline $9 / 5$ & $84^{\circ}$ & 551 \\
\hline $9 / 6$ & 850 & 550 \\
\hline $9 / 7$ & 853 & 553 \\
\hline $9 / 8$ & $82^{\circ}$ & 552 \\
\hline $9 / 9$ & 844 & 534 \\
\hline
\end{tabular}

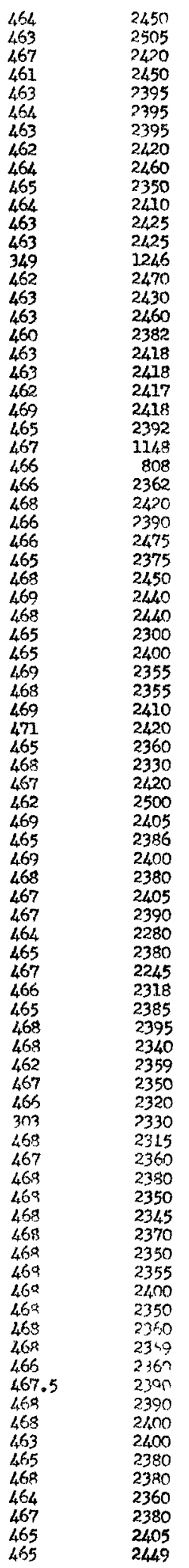

\begin{tabular}{|c|c|c|}
\hline 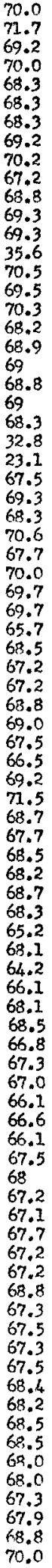 & 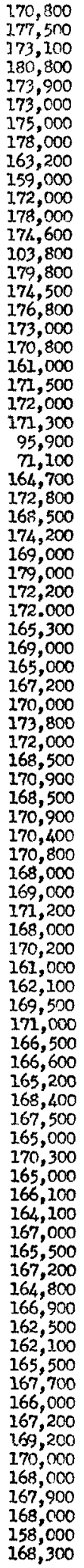 & 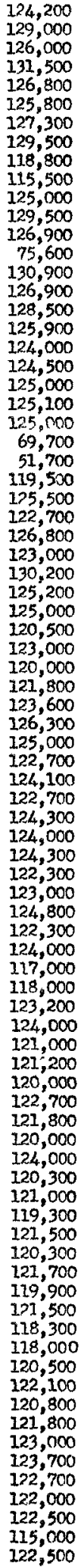 \\
\hline
\end{tabular}

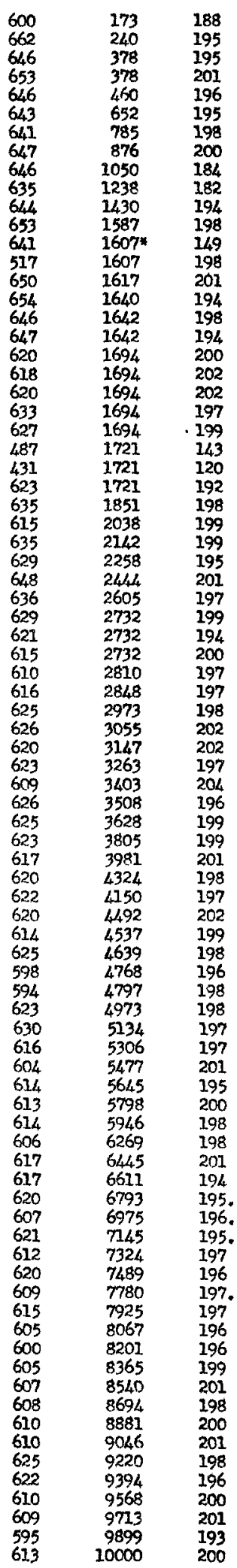




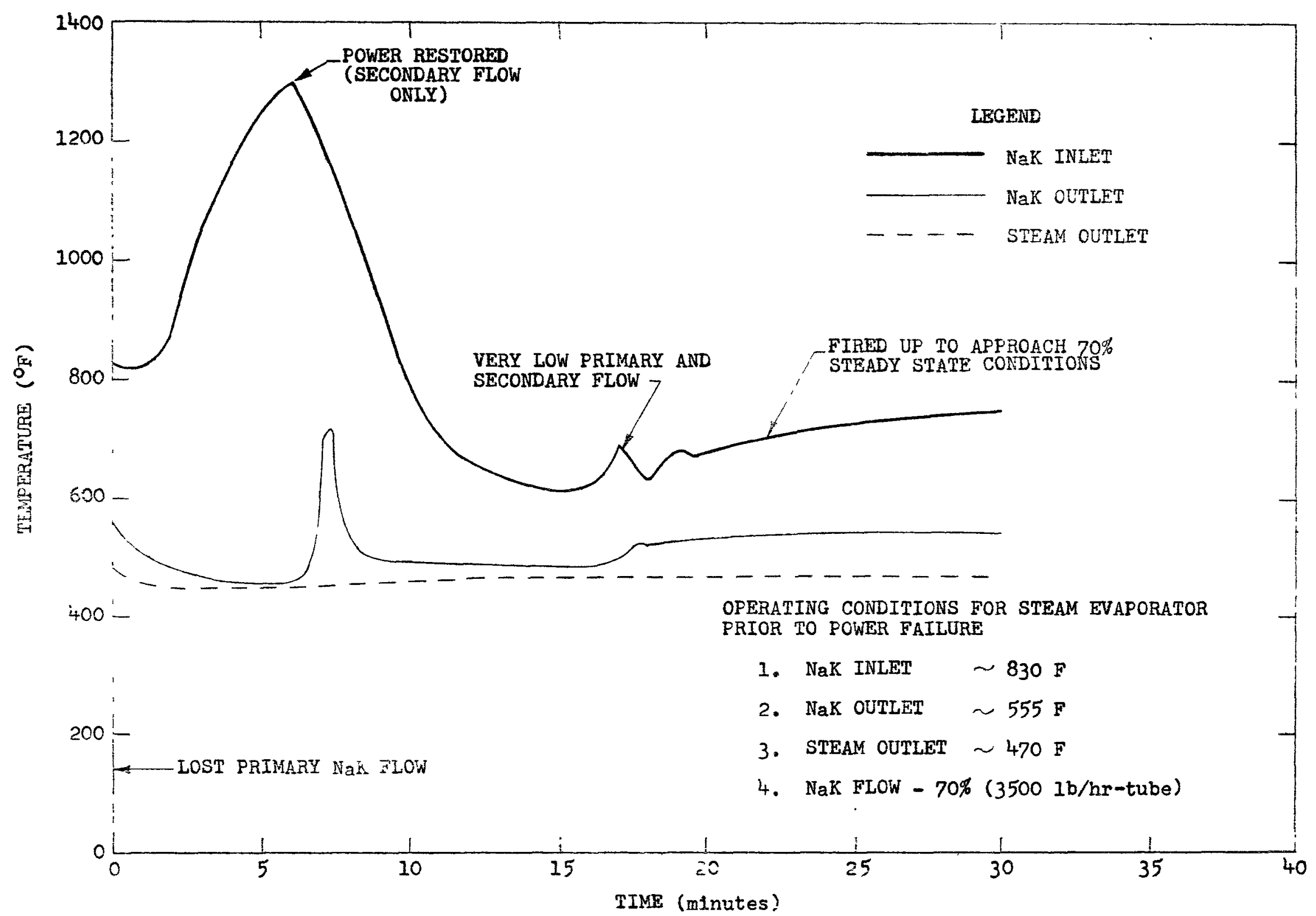

Fig. 14 - High NaK Temperature Incident 6/19/56 Two Tube Steam Evaporitor No. 3 
5. FINAL STEADY STATE OPERATION

\subsection{Operations}

Upon completion of the cyclic program, steady state heat transfer data was obtained with the evaporator operating at $70 \%, 60 \%, 40 \%, 25 \%$ and finally $20 \%$ of full power. In collecting data at each power level, the unit was first allowed to attain steady state conditions for an hour, then three readings were taken at half hour intervals.

\subsection{Results}

The overall heat transfer coefficient, $U$, was calculated over the range of power levels, and shown in Table 8 . These have been plotted and are shown in Fig. 15, along with a copy of the curve of Fig. 7 for easy comparison. The effect of the thermal cycling upon the performance of the evaporator was a lowering of the heat transfer coefficient rather uniformIy over the whole range of operation. U for $30 \%$ power was lowered from 480 to about $443 \mathrm{Btu} / \mathrm{hr}-\mathrm{sq}$ ft- $\mathrm{F}$ while at $70 \%$ power was lowered from 660 to about $610 \mathrm{Btu} / \mathrm{hr}-\mathrm{sq}$ ftm ${ }^{\circ} \mathrm{F}$.

After this series of data was obtained, the unit was operated for an additional day at low power in anticipation of further testing instructions. On Sept. 13, 1956, no further word having been received, the furnace was secured and the steam system blanketed with dry nitrogen at 25 psig. 
Table 8 - Heat Transfer Data At Different Power Levels After Completion of Cycling Program

\begin{tabular}{|c|c|c|c|c|c|c|c|c|c|c|}
\hline \multirow{2}{*}{$\begin{array}{l}\text { Date } \\
1956 \\
\end{array}$} & \multirow[b]{2}{*}{ Hour } & \multicolumn{2}{|c|}{$\begin{array}{l}\text { Evaporator } \\
\text { NaK Temp }{ }^{\circ}\end{array}$} & \multirow{2}{*}{$\begin{array}{l}\text { Steam } \\
\text { Temp. OF }\end{array}$} & \multirow{2}{*}{$\begin{array}{r}\mathrm{NaK} \\
\mathrm{Ib} / \mathrm{hr}-\text { tube } \\
\end{array}$} & \multirow{2}{*}{$\begin{array}{l}\text { Flow } \\
\% \text { of } 3500 \\
1 \mathrm{~b} / \mathrm{hr} \text {-tube } \\
\end{array}$} & \multirow{2}{*}{$\begin{array}{l}Q=B t u / \\
h r-t u b e\end{array}$} & \multirow{2}{*}{$\begin{array}{l}\text { Heat Flux } \\
Q / A=B t u / h r \\
-s q \text { ft-tube }\end{array}$} & \multirow[b]{2}{*}{$\mathrm{U}$} & \multirow{2}{*}{$\begin{array}{l}\triangle \mathrm{T} \\
\mathrm{O}_{\mathrm{F}} \\
\end{array}$} \\
\hline & & Inlet & Outlet & & & & & & & \\
\hline $9 / 9$ & $4: 15 \mathrm{PM}$ & 844 & 554 & 465 & 2455 & 70.2 & 168,800 & 122,900 & 625 & 200 \\
\hline \multirow[t]{11}{*}{$9 / 10$} & $1: 30 \mathrm{AM}$ & 853 & 557 & 466 & 2450 & 70 & 172,000 & 125,000 & 613 & 204.4 \\
\hline & $9: 15 \mathrm{AM}$ & 852 & 554 & 465 & 2400 & 68.5 & 169,600 & 123,500 & 610 & 202.6 \\
\hline & $11: 00 \mathrm{AM}$ & 844 & 553 & 465 & 2413 & 68.9 & $166,600^{\circ}$ & 121,200 & 609 & 199 \\
\hline & $12: 30 \mathrm{PM}$ & 842 & 541 & 464 & 2065 & 59 & 147,600 & 107,600 & 569 & 189 \\
\hline & $1: 30 \mathrm{PM}$ & 849 & 542 & 465 & 2065 & 59 & 150,200 & $109 ; 500$ & 574 & 191 \\
\hline & $2: 30 \mathrm{PM}$ & 850 & 542 & 464 & 2060 & 58.8 & 150,600 & 109,800 & 570 & 192.5 \\
\hline & $4: 00 \mathrm{PM}$ & 846 & 513 & 465 & 1388 & 39.6 & 110,200 & 80,300 & 499 & 160.7 \\
\hline & $5: 00 \mathrm{PM}$ & 846 & 513 & $465^{\prime}$ & 1389 & 39.7 & 110,500 & 80,400 & 500 & 160.8 \\
\hline & $6: 00 \mathrm{PM}$ & 850 & 513 & 464 & 1388 & 39.65 & 111,800 & 81,300 & 497 & 163.5 \\
\hline & $9: 30 \mathrm{PM}$ & 849 & 492 & 465 & 864 & 24.6 & 74,000 & 53,800 & 400 & 134.4 \\
\hline & $10: 30 \mathrm{PM}$ & 848 & 494 & 465 & 865 & 24.7 & 73,250 & 53,400 & 388 & 137.3 \\
\hline \multirow[t]{3}{*}{$9 / 11$} & $3: 00 \mathrm{AM}$ & 847 & 482 & 465 & 697 & 19.9 & 61,110 & 44,500 & 373 & 119.2 \\
\hline & $7: 30 \mathrm{AM}$ & 851 & 481 & 464 & 692 & 19.75 & 61,400 & 44,600 & 377 & 118.3 \\
\hline & $11: 00 \mathrm{AM}$ & 852 & 490 & 464 & 857 & 24.5 & 74.400 & 54,100 & 404 & 134 \\
\hline $9 / 12$ & & 851 & 500 & 465 & 1061 & 30.3 & 89,000 & 64,800 & 443 & 146.2 \\
\hline $9 / 13$ & & 850 & 500 & 464 & 1060 & 30.3 & 88,880 & 64,590 & 443 & 145.9 \\
\hline
\end{tabular}




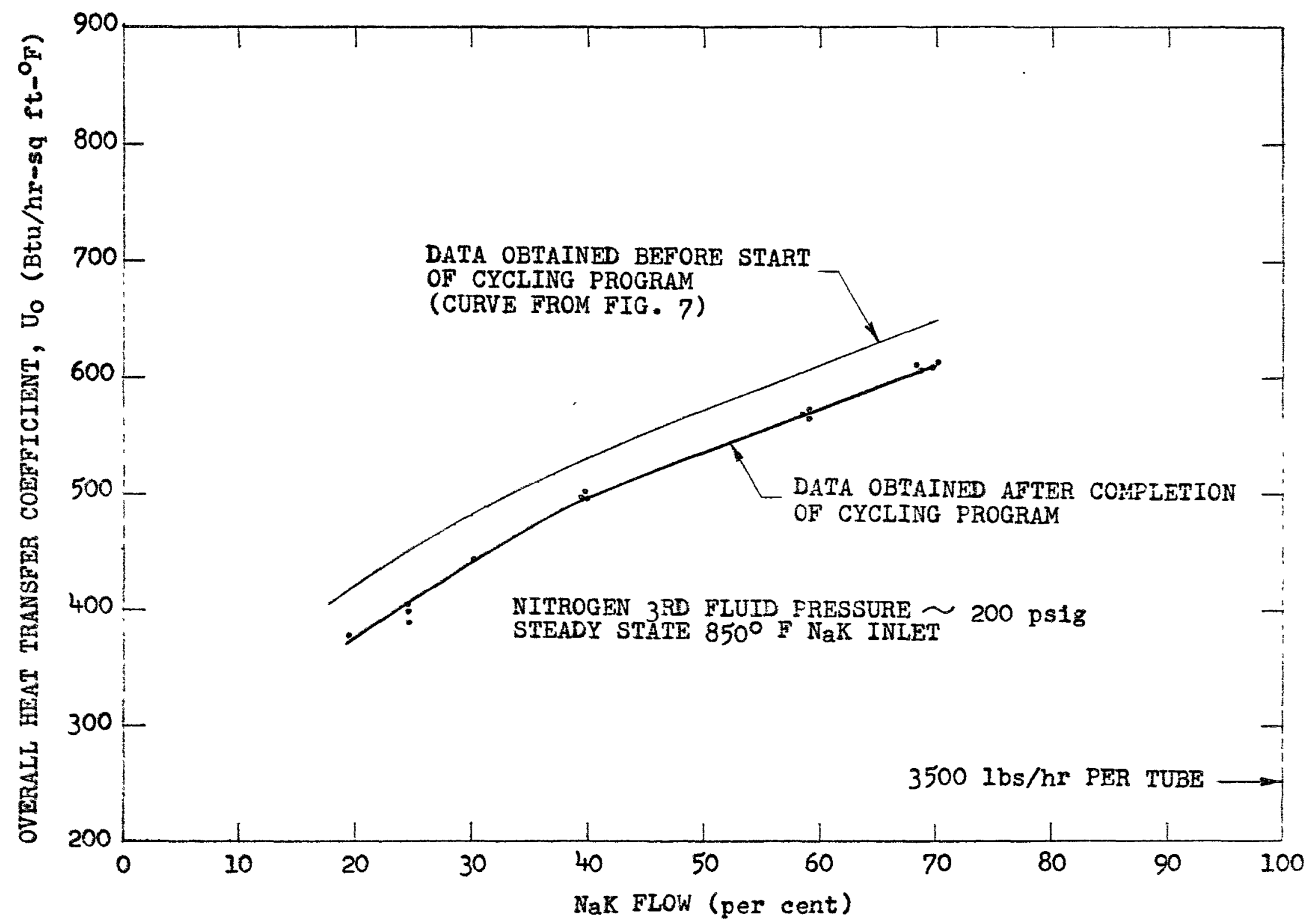

Fig. 15 - Heat Transfer Performance of Duplex Tube at Several Power Levels of $G-R$ Two Tube Evaporator No. 3 Before and After Cycling Program 


\section{EVAPORATOR WATER}

Inasmuch as the purpose of this experiment was to test the choice of the material of construction and the operating conditions as a means for minimizing the effects of chloride stress corrosion, a strict boiler water quality control was maintained throughout the program.

Before charging with water, the steam system was cleaned by circulating a solution of $15 \% \mathrm{HCI}$ and $0.2 \%$ inhibitor at $150 \mathrm{~F}$ for several hours, then the system was rinsed with demineralized water, purged with nitrogen and vacuum dried. The water was charged into an evacuated system and during all times when not steaming, the system was blanketed with nitrogen.

Table 9 shows the water analyses determined throughout the program and the prescribed specifications. A point-to-point record of each analysis over the entire test program is shown inig. 16.

Table 9 - Boiler Water Analysis and Specifications for Two Tube Steam Generator \#3

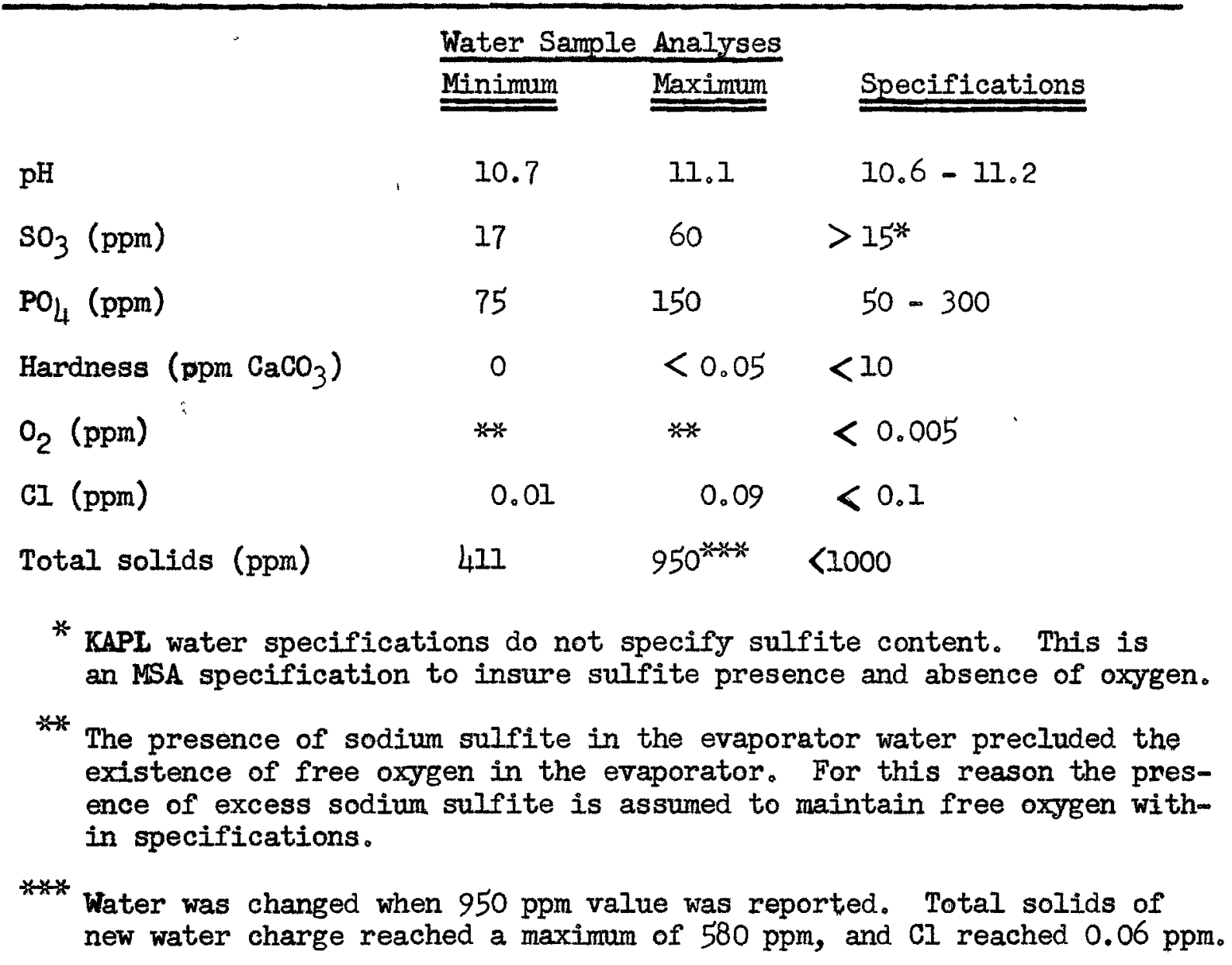



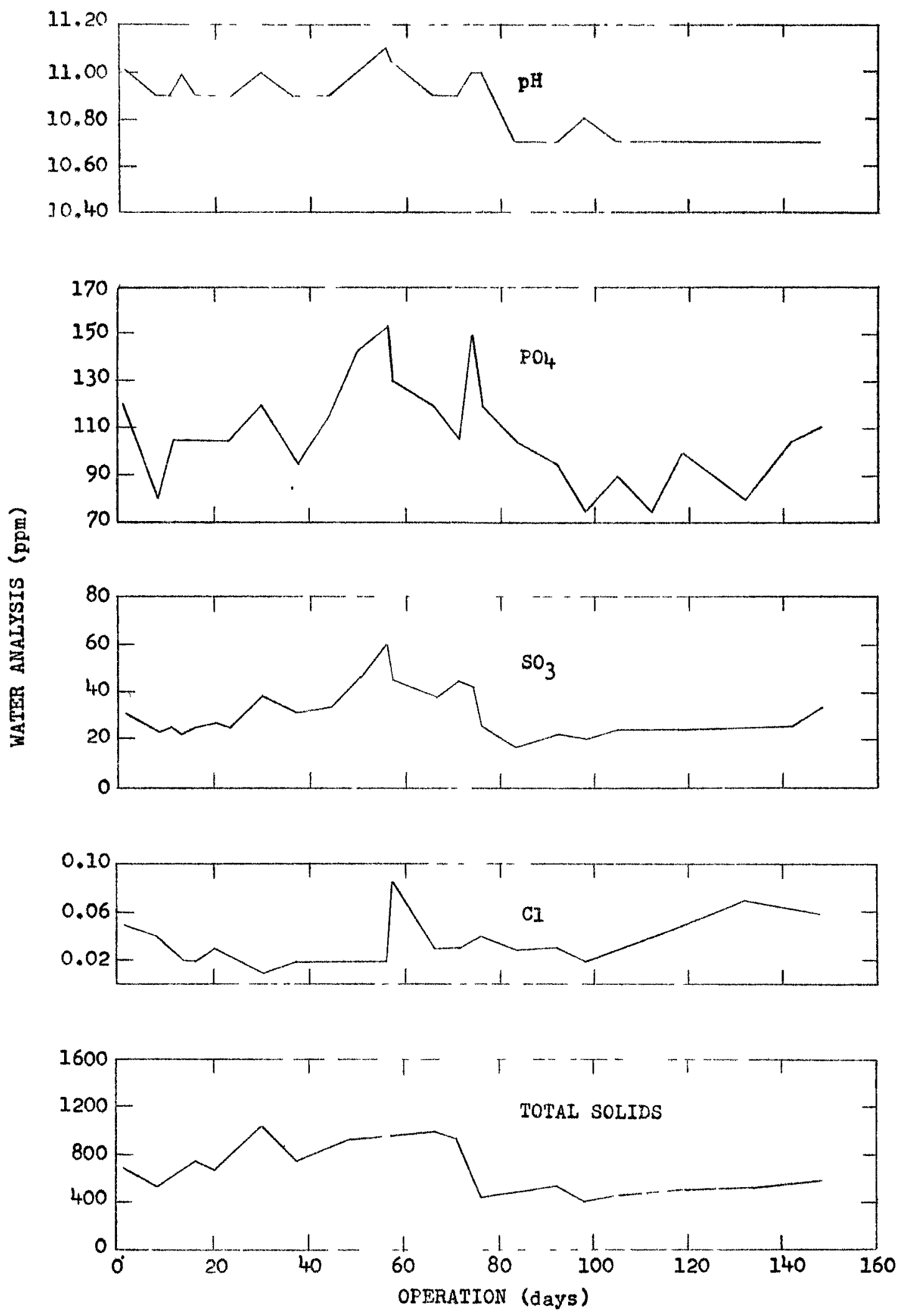

F1g. 16 - Water Analysis During Operation of

G-R Two Tube Steam Evaporator No. 3 


\section{OVERALL RESULTS AND DISCUSSIONS}

\subsection{Comparison of Data on This Test}

The performance of the evaporator was appreciably higher ( $\sim 28 \%$ ) when helium instead of nitrogen was used as the third fluid in the annuli spaces of the duplex tubes at 200 psig. A comparison at $60 \%$ and $30 \% \mathrm{NaK}$ flow conditions indicates that $U$ will be 765 and $633 \mathrm{Btu} / \mathrm{hr}-\mathrm{sq}$ ft-oF respectively for the helium condition as compared to 602 and 492 respectiveIy for the nitrogen condition. These differences are most likely due to the higher thermal conductivity of helium over that of nitrogen ( $\sim 0.5$ to I).

The increase in $U$ with an increase in the third fluid gas pressure is very definite at low pressures up to about 2 atmospheres (see Fig. 6, p.17). This is expected as the number of molecules of gas in the third fluid system to conduct heat is increased. However, between 2 and 20 atmospheres there is very little change in overall $U$, presumably because in this pressure range, a change in pressure has very little effect on the thermal conductivity of the gas, and the increase pressure may cause a loss of mechanical bond between the outer and inner tubes.

A complete summation of the operating time of the evaporator at different conditions is given in Table 10.

There was very little change in $U$ during the initial steady state studies even though tests were made over a 6 week period.

During the cyclic program $\mathrm{J}$ dropped from 660 to $610 \mathrm{Btu} / \mathrm{hr}-\mathrm{sq}$ ft- ${ }^{\circ} \mathrm{F}$, a 7.5\% drop, with $74 \%$ of the drop occurring the first $25 \%$ of the cyclic test (see Fig. 8, p 22). Fig. 15 shows that there seems to be a break in the $U$ vs per cent $\mathrm{NaK}$ flow curve at $40 \%$ power following these cyclic tests. These findings may be due to a slight separation of the mechanical bond due to stress relieving and would be exemplified at low power loads. As the load is decreased the inner tube becomes more nearly equal to the outer tube in temperature and thus contracts more. At loads below $40 \%$ the differences in expansion may just cause a gas gap to appear between the two tubes. Above $40 \%$ loads, the two tubes may again come into contact and further expansion of the inner tube with respect to the outer tube with increased temperature difference would contribute very little to added heat transfer.

The present evaporator is to have the inner tubes re-expanded and the test continued to see if the original operating efficiency can be recovered.

\subsection{Comparison of This Evaporator With Two Made of Stainless Steel}

MSA Technical Report No. $28^{l}$ describes the tests and results performed on two previously built evaporators, the first made of Type 347

1 Andrews, R. C., King, E. C., Effects of Thermal Cycling and Chloride Corrosion on Stainless Steel Steam Generators, Technical Report 28, (Contract NObs-65426) Mine Safety Appliances Co., July 30, 1954. 
Table 10 - Operational History of Two Tube Generator No. 3 500 psig Steam - 200 psig $\mathrm{N}_{2}$ Third Fluid

\begin{tabular}{|c|c|c|c|c|}
\hline \multirow[b]{2}{*}{$\begin{array}{l}\text { Operating } \\
\text { Condition }\end{array}$} & \multicolumn{3}{|c|}{ Operating Time, Hours } & \multirow[b]{2}{*}{ Total } \\
\hline & $\begin{array}{l}4 / 16 / 56 \\
\text { to } \\
5 / 31 / 56 \\
\text { Initial } \\
\text { Steady } \\
\text { State } \\
\end{array}$ & $\begin{array}{c}6 / 1 / 56 \\
\text { to } \\
9 / 9 / 56 \\
\text { Cycling } \\
\text { Program } \\
\end{array}$ & $\begin{array}{c}9 / 9 / 56 \\
\text { to } \\
9 / 13 / 56 \\
\text { Final } \\
\text { steady } \\
\text { State } \\
\end{array}$ & \\
\hline $\begin{array}{r}70 \% \text { flow (steady } \\
\text { state) }\end{array}$ & & 350 & 35 & 385 \\
\hline $\begin{array}{r}60 \% \text { flow (steady } \\
\text { state) }\end{array}$ & & & 3 & 3 \\
\hline $\begin{array}{r}40 \% \text { flow (steady } \\
\text { state) }\end{array}$ & & & 3 & 3 \\
\hline $\begin{array}{r}30 \% \text { flow (steady } \\
\text { state) }\end{array}$ & & 301 & 48 & 349 \\
\hline $\begin{array}{r}25 \% \text { flow (steady } \\
\text { state) }\end{array}$ & & & 7 & 7 \\
\hline $\begin{array}{r}20 \% \text { flow (steady } \\
\text { state) }\end{array}$ & & & 10 & 10 \\
\hline Cycling & & 1334 & & 1334 \\
\hline $\begin{array}{l}\text { Changing of power } \\
\text { level (estimate) }\end{array}$ & & 72 & & 72 \\
\hline Total Hours Steaming & 792 & 2057 & 106 & 2955 \\
\hline Down Time & 294 & 392 & & 686 \\
\hline$*$ Based on $100 \%=3500$ & $0 \mathrm{Ibs} / \mathrm{hr}-$ & & & \\
\hline
\end{tabular}


S S (No. I) and the second made of Type 304 (No. 2). The cycling program for evaporators No. 1 and No. 2 are found in Tables 11 and 12 . A tabulation of the important data for all these units is found in Table 13. Fig. 17 is a comparison of the performance of the 3 evaporators. Evaporators No. 1 and 2 were tested using synthetic sea water in the feed water system while S2G water was used in No. 3 .

Evaporator No. 2 failed, presumably by chloride stress corrosion. Bvaporator No. 1, Type $347 \mathrm{~s} \mathrm{~s}$, did not show the percentage decrease in $\mathrm{U}$ that evaporator No. 2 did. However, whereas the $\mathrm{U}$ calculated for evaporator No. 3 at $70 \%$ power was $610 \mathrm{Btu} / \mathrm{hr}-\mathrm{sq}$ ft- ${ }^{\circ} \mathrm{F}$ at completion of the test program, U for evaporator No. 1 at the start was only 575 . With helium as the third fluid in evaporator No. 3 the coefficient was even higher. Evaporator No. 3 did show a greater drop in coefficient than did No. 1 but the test was for 10,000 cycles whereas No. I was only 1,000 cycles. If only the first 1,000 cycles of operation of evaporator No. 3 are considered, then that unit suffered a drop of only $3 \%$ in $U$. This compares well with the $2 \%$ drop in $U$ experienced with evaporator No. I during 1,000 cycles.

It should be expected that thermal cycling would have the effect of accelerating creep. It remains for metallographic examination to determine the extent of any creep, or other strain in the No. 3 evaporator, and to determine if any structural weakness exists. 
Table 11 - Gyclic Program For Steam Generator No. 1

\begin{tabular}{|c|c|c|c|c|c|c|}
\hline $\begin{array}{l}\text { Cycle } \\
\text { No. }\end{array}$ & $\begin{array}{l}\text { Inlet } \mathrm{NaK} \\
\text { Temp. } \\
\text { (OF) }\end{array}$ & $\begin{array}{l}\text { Time } \\
\text { Sequences } \\
\text { (Min) }\end{array}$ & $\begin{array}{c}\text { NaK Flow } \\
\% \text { of } \\
7000 \mathrm{lbs} / \mathrm{hr}\end{array}$ & $\begin{array}{l}\text { Steam } \\
\text { Pressure } \\
\text { Psig } \\
\end{array}$ & $\begin{array}{l}\text { Cycles } \\
\text { Required }\end{array}$ & Method of Control \\
\hline 1 & $\begin{array}{l}820 \\
700 \\
820\end{array}$ & $\begin{array}{l}\frac{1}{2} \text { up } \\
\frac{1}{1} 4 \frac{1}{2} \text { hold } \\
\frac{1}{2} \text { down }\end{array}$ & 71.5 & 500 & 1000 & $\begin{array}{l}\text { Reduce primary NaK flow } \\
\text { rate to } 28.2 \% \text { or normal. } \\
\text { Cool with secondary loop. }\end{array}$ \\
\hline 2 & $\begin{array}{l}850 \\
650 \\
850\end{array}$ & $\begin{array}{l}1 / 30 \text { down } \\
15 \text { hold } \\
3 \text { up }\end{array}$ & 71.5 & 500 & 20 & $\begin{array}{l}\text { Primary NaK flow rate } \\
\text { constant. Cool with } \\
\text { secondary loop. }\end{array}$ \\
\hline 3 & $\begin{array}{r}850 \\
1400 \\
850\end{array}$ & $\begin{array}{l}5 \text { up } \\
15 \text { hold } \\
10 \text { down }\end{array}$ & 71.5 & 500 & 4 & $\begin{array}{l}\text { Reduce primary } \mathrm{NaK} \\
\text { flow rate. }\end{array}$ \\
\hline 4 & $\begin{array}{r}850 \\
1080 \\
850\end{array}$ & $\begin{array}{l}1 \text { up } \\
15 \text { hold } \\
3 \text { down }\end{array}$ & 71.5 & 500 & 4 & $\begin{array}{l}\text { Reduce primary NaK flow } \\
\text { rate. }\end{array}$ \\
\hline 5 & $\begin{array}{r}850 \\
1400 \\
850\end{array}$ & $\begin{array}{l}1 \text { up } \\
15 \text { hold } \\
5 \text { down }\end{array}$ & 71.5 & 500 & 4 & $\begin{array}{l}\text { Hold inlet NaK initial } \\
\text { condition with secondary } \\
\text { loop, then turn off } \\
\text { secondary loop and re- } \\
\text { duce primary loop flow } \\
\text { rate simultaneously. }\end{array}$ \\
\hline
\end{tabular}


Table 12 - Cyclic Program for Steam Generator No. 2

\begin{tabular}{|c|c|c|c|c|c|c|}
\hline $\begin{array}{l}\text { Cycle } \\
\text { No. }\end{array}$ & $\begin{array}{l}\text { Inlet } \mathrm{NaK} \\
\text { Temp. } \\
\left({ }^{\circ} \mathrm{F}\right) \\
\end{array}$ & $\begin{array}{c}\text { Time } \\
\text { Sequence } \\
\text { (Min) } \\
\end{array}$ & $\begin{array}{c}\text { NaK Flow } \\
\% \text { of } \\
7000 \mathrm{lb} / \mathrm{hr} \\
\end{array}$ & $\begin{array}{l}\text { Steam } \\
\text { Pressure } \\
\text { Psig } \\
\end{array}$ & $\begin{array}{c}\text { Cycles } \\
\text { Required } \\
\end{array}$ & Method of Control \\
\hline 1 & $\begin{array}{l}850 \\
946 \\
850\end{array}$ & $\begin{array}{l}\frac{1}{2} \text { up } \\
\frac{1}{1} 4 \frac{1}{2} \text { hold } \\
3 \text { down }\end{array}$ & 63.8 & 500 & 100 & $\begin{array}{l}\text { Reduce primary NaK flow to } \\
\text { obtain temp. Secondary loop } \\
\text { turned off. }\end{array}$ \\
\hline 2 & $\begin{array}{l}850 \\
740 \\
850\end{array}$ & $\begin{array}{l}\frac{1}{2} \text { up } \\
\frac{1}{1} 4 \frac{1}{2} \text { hold } \\
\frac{1}{2} \text { down }\end{array}$ & 63.8 & 500 & 2500 & $\begin{array}{l}\text { Primary NaK flow reduced to } \\
\text { I } 2 \% \text {. Cool with secondary } \\
\text { loop. }\end{array}$ \\
\hline 3 & $\begin{array}{r}850 \\
1750 \\
850\end{array}$ & $\begin{array}{l}2 \text { up } \\
5 \text { hold } \\
5 \text { down }\end{array}$ & 63.8 & 500 & 100 & $\begin{array}{l}\text { Reduce primary NaK flow to } \\
\text { obtain temp. Secondary loop } \\
\text { turned off. }\end{array}$ \\
\hline 4 & $\begin{array}{l}850 \\
650 \\
850\end{array}$ & $\begin{array}{l}1 / 30 \text { down } \\
5 \text { hold } \\
3 \text { up }\end{array}$ & 63.8 & 500 & 100 & $\begin{array}{l}\text { Primary NaK flow constant. } \\
\text { Cool with secondary loop. }\end{array}$ \\
\hline 5 & $\begin{array}{l}850 \\
470 \\
850\end{array}$ & $\begin{array}{l}10 \text { down } \\
5 \text { hold } \\
1 \frac{1}{2} \text { up }\end{array}$ & $\begin{array}{r}63.8 \\
3.2\end{array}$ & 500 & 100 & $\begin{array}{l}\text { Primary flow reduced to } \\
\text { 5\%. Cool with secondary } \\
\text { loop. Temp. and flow re- } \\
\text { turned within prescribed } \\
\text { time. }\end{array}$ \\
\hline
\end{tabular}


Table 13 - Operating Conditions of Evaporators No, 1, 2 and 3

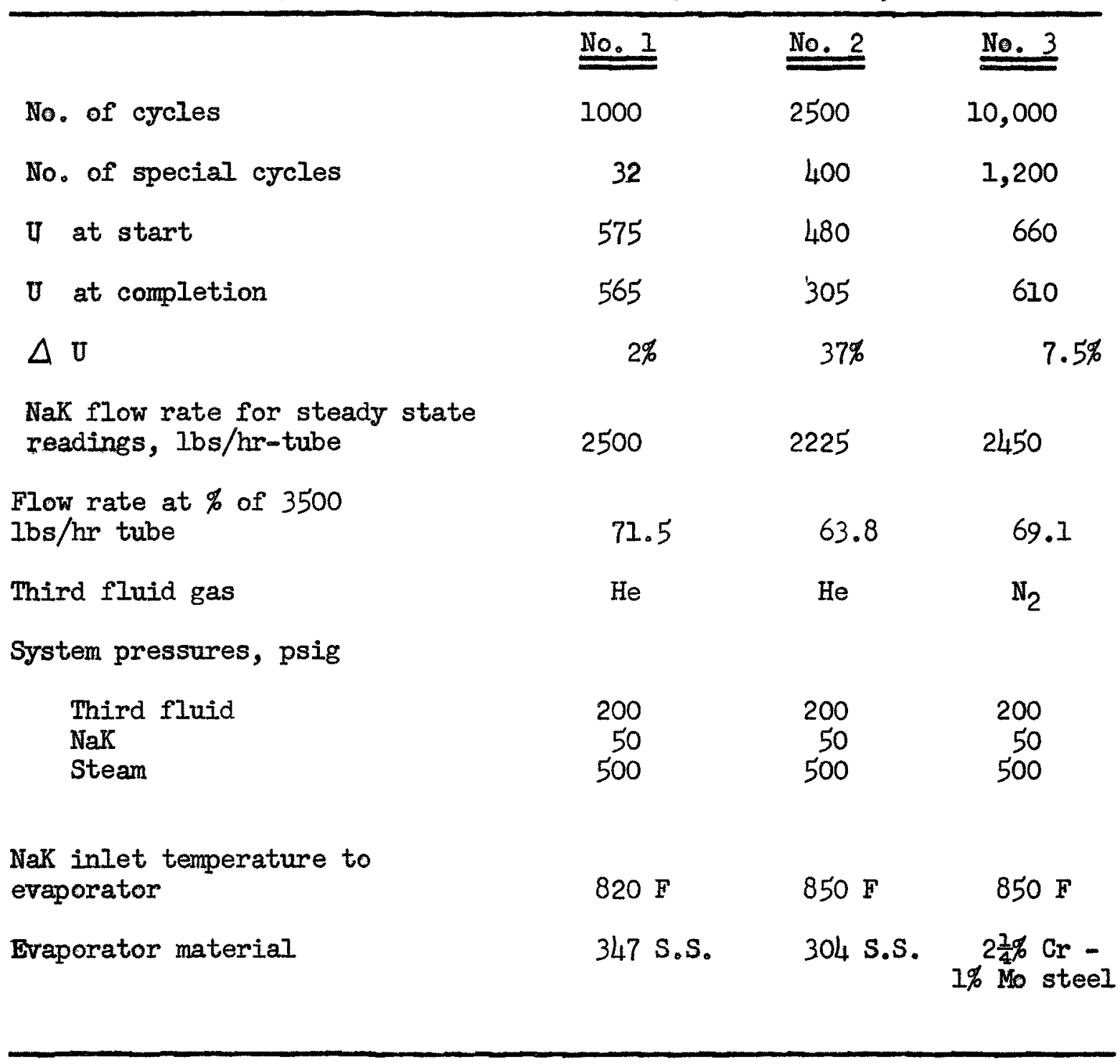




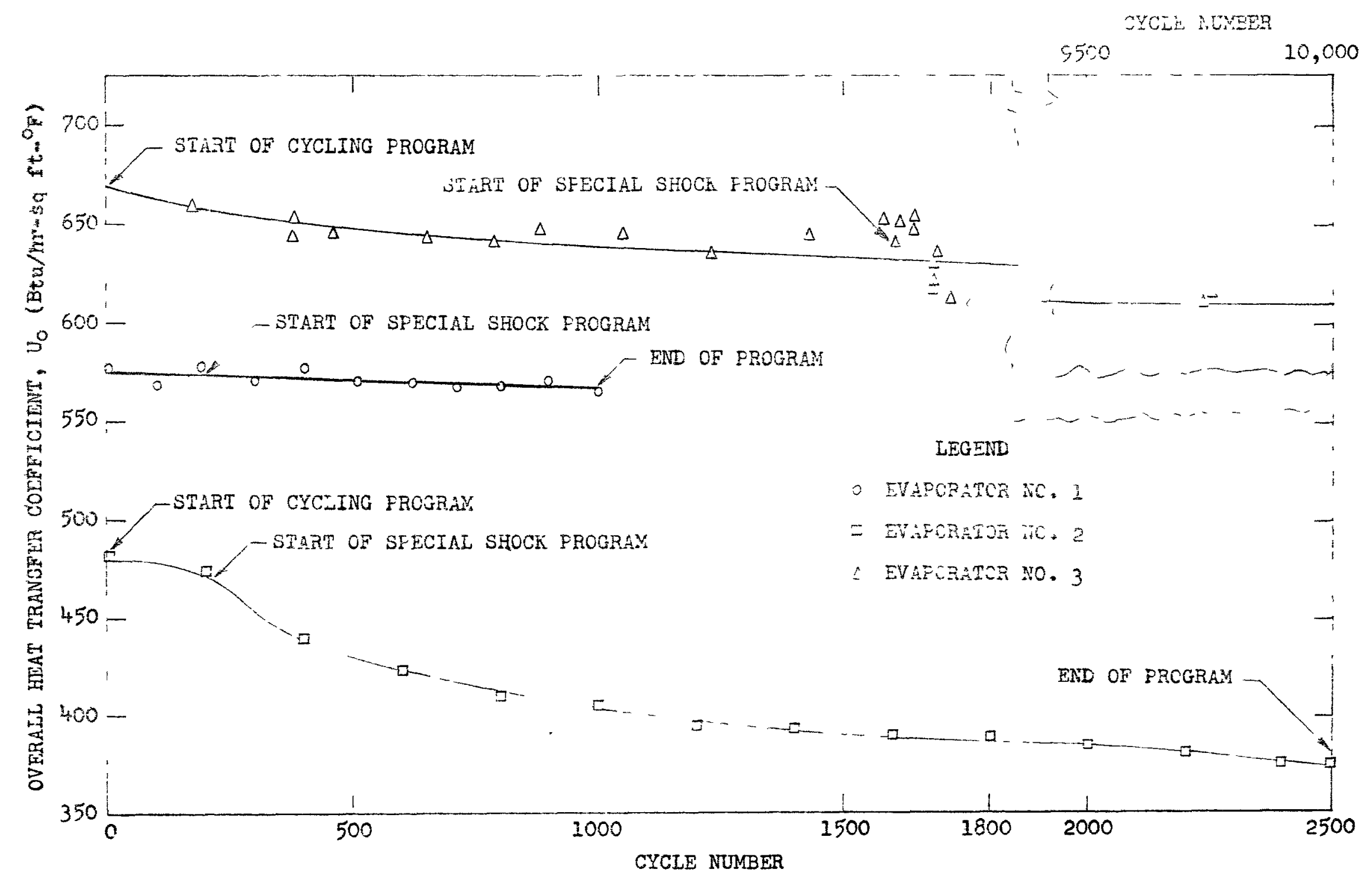

Fig. 17 - Comparison of G-R Two Tube Fvaporators 


\section{CONCLUSIONS}

The $2 \frac{1}{4} \% \mathrm{Cr}$ - 1\% Mo steel evaporator compared favorably with the type 347 stainless steel evaporator and out-performed the type 304 stainless steel evaporator which failed before completion of the testing program. If metallographic tests show that the evaporator is structurally sound, then advantages to be had in using this latest design include cheapness of material of construction, higher thermal conductivity and freedom from dependence on chloride free water as the alloy is not as susceptible to chloride stress corrosion.

Advantages, such as material cost and space may be had by using helium in the third fluid system rather than nitrogen, since a unit using helium would reduce the size by $\frac{2}{4}$ for a given rated power output. 